Pacific Journal of Mathematics

ENDOSCOPIC GROUPS AND BASE CHANGE ChR 


\title{
ENDOSCOPIC GROUPS AND BASE CHANGE $\mathbf{C} / \mathbf{R}$
}

\author{
D. SHELSTAD
}

We consider a real reductive group $G$ with complex points $G(\mathrm{C})$, Galois automorphism $\sigma$, and real points $G(\mathbf{R})=\{g \in G(\mathbf{C}): \sigma(g)=g\}$. In general, an irreducible admissible representation $\Pi$ of $G(\mathrm{C})$ equivalent to its Galois conjugate $\Pi \circ \sigma$ need not be a lift from $G(\mathbf{R})$, even if $G$ is quasi-split over $\mathbf{R}$. Following the results of $L$-indistinguishability we might expect this phenomenon to be related to the fact that $\sigma$-twisted conjugacy on $G(\mathrm{C})$ need not be "stable", and therefore attempt to match the various "unstable" combinations of $\sigma$-twisted orbital integrals on $G(\mathrm{C})$ with stable orbital integrals on certain groups $H(\mathrm{R})$. The principle of functoriality in the $L$-group would then suggest, with reservations in the nontempered case, a relation between the $\sigma$-twisted characters of representations of $G(C)$ fixed up to equivalence by $\sigma$ and the "dual lifts" to $G(\mathrm{C})$ of stable characters on the groups $H(\mathbf{R})$.

In this paper we define the relevant groups $H \ldots$ they turn out to be the endoscopic groups from $L$-indistinguishability... and prove a matching theorem for orbital integrals. As a preliminary to the proposed dual liftings of characters we also study the "factoring" of Galois-invariant Langlands parameters for $G(\mathrm{C})$.

1. Introduction. We begin with two simple examples. Let $G(\mathbf{C})=$ $\mathbf{C}^{x}$ and $\sigma(z)=\bar{z}^{-1}, z \in \mathbf{C}^{x}$, so that $G(\mathbf{R})=\{g \in G(\mathbf{C}): \sigma(g)=g\}$ is the unit circle in $\mathbf{C}^{x}$. A quasicharacter on $\mathbf{C}^{x}$ fixed by $\sigma$, i.e., trivial on the positive reals, need not be of the form $z \rightarrow \chi(z \sigma(z))=\chi(z / \bar{z})$, with $\chi$ a character on the unit circle. At the same time $z \in \mathbf{C}^{x}$ is stably $\sigma$-conjugate to $-z$, but not $\sigma$-conjugate (see [Sh6] for definitions). Let $f \in C_{c}^{\infty}\left(\mathbf{C}^{x}\right)$ and write $f(r, \theta)$ for $f\left(r e^{i \theta}\right)$. Set $H_{1}=H_{2}=G$, so that $H_{1}(\mathbf{R})=S^{1}$. Let

$$
f_{1}\left(e^{i \theta}\right)=\frac{1}{2} \int_{0}^{\infty}(f(r, \theta / 2)+f(r, \theta / 2+\pi)) d r / r
$$

and

$$
f_{2}\left(e^{i \theta}\right)=\frac{1}{2} e^{i \theta / 2} \int_{0}^{\infty}(f(r, \theta / 2)-f(r, \theta / 2+\pi)) d r / r
$$

for $-\pi<\theta<\pi$. Then both $f_{1}$ and $f_{2}$ extend smoothly to $S^{1}$. If $\chi$ is a character on $S^{1}$ then $f \rightarrow \int_{-\pi}^{\pi} \chi\left(e^{i \theta}\right) f_{1}\left(e^{i \theta}\right) d \theta$ is a distribution on $\mathbf{C}^{x}$ representing the usual lift of $\chi$ to $G(\mathbf{C})$, i.e., representing the quasicharacter $z \rightarrow \chi(z \sigma(z))$. On the other hand, $f \rightarrow \int_{-\pi}^{\pi} \chi\left(e^{i \theta}\right) f_{2}\left(e^{i \theta}\right) d \theta$ lifts $\chi$ to the quasicharacter $z=r e^{i \theta} \rightarrow \chi(z \sigma(z)) e^{i \theta}$. We have therefore recovered the remaining Galois-invariant quasicharacters on $\mathbf{C}^{x}$. 
For a general group, however, there are difficulties more akin to those for $L$-indistinguishability. Consider $G=\mathrm{SL}_{2}$. Let

$$
H(\mathbf{R})=\left\{r(\theta)=\left[\begin{array}{cc}
\cos \theta & \sin \theta \\
-\sin \theta & \cos \theta
\end{array}\right]\right\} .
$$

Note that if $\theta \neq 0(\bmod \pi)$ then $r(\theta)$ and $r(\theta+\pi)$ are stably $\sigma$-conjugate in $G(\mathbf{C})$ but not $\sigma$-conjugate (see [Sh6, Lemma 2.5.2]). For $f \in C_{c}^{\infty}\left(\mathrm{SL}_{2}(\mathbf{C})\right.$ ), define

$$
f_{H}(r(\theta))=e^{i \theta / 2}\left(e^{i \theta}-e^{-i \theta}\right)\left(\Phi_{f}^{\sigma}(\theta / 2)+\Phi_{f}^{\sigma}(\theta / 2+\pi)\right),
$$

for $-\pi<\theta<\pi$, where

$$
\Phi_{f}^{\sigma}(\theta)=\int_{G(\mathbf{C}) / H(\mathbf{R})} f\left(\sigma(g) r(\theta) g^{-1}\right) \frac{d g}{d \theta},
$$

$d g$ denoting a Haar measure on $G(\mathbf{C})=\mathrm{SL}_{2}(\mathbf{C})$. It can be shown that $f_{H}$ extends to a $C^{\infty}$ function on $H(\mathbf{R})$. Then $f \rightarrow \int_{H(\mathbf{R})} \chi f_{H}$ is a distribution on $\mathrm{SL}_{2}(\mathrm{C})$ (see [Sh6, §5.4] for an explicit formula). L. Clozel has shown that this distribution is, up to a constant, the twisted character of a Galois-fixed equivalence class of representations of $\mathrm{SL}_{2}(\mathbf{C})$. It is easily verified that all such classes of (irreducible, admissible) representations of $\mathrm{SL}_{2}(\mathbf{C})$ which are not lifts from $\mathrm{SL}_{2}(\mathbf{R})$ are lifts in this way.

Returning to the general problem, we find it convenient to consider $G(\mathbf{C})$ as the group of real points on a group $\tilde{G}$, and $\sigma$ as the restriction to $\tilde{G}(\mathbf{R})$ of an algebraic automorphism $\alpha$ of $\tilde{G}$ (cf. $\S 2$ ). Also, since $(\tilde{G}, \alpha)$ is our starting point, rather than $G$ itself, we may as well assume that $G$ is quasi-split over $\mathbf{R}$.

In this paper we will be concerned with the matchings for $\alpha$-twisted orbital integrals on $\tilde{G}(\mathbf{R})$; this includes the problem of determining what it is they should match. Theorem 7.1 is our main result, and $\S \S 2$ to 6 are preparation for it. Also, as both a check on our definitions and a preliminary to the proposed dual liftings, we will consider the question of "factoring" Galois-invariant Langlands parameters for $G(\mathbf{C})$ or, equivalently [L1] $\alpha$-invariant parameters for $\tilde{G}(\mathbf{R})$. Theorem 8.1 is the main result.

In [Sh6] we started a study of the matching problem for $\alpha$-twisted orbital integrals. We found that, despite various "technical" difficulties, the jump formulas for twisted orbital integrals on $\tilde{G}(\mathbf{R})$ are closed related to those for ordinary orbital integrals on $G(\mathbf{R})$. Making convenient technical assumptions, we then put together a matching theorem involving the endoscopic groups from $L$-indistinguishability. In this paper we start afresh, making none of the technical assumptions of [Sh6]. We first define 
the notion of endoscopic group for $(\tilde{G}, \alpha)$. This turns out to be the same as the notion of endoscopic group in $L$-indistinguishability [L3], [Sh4]. However, there is new information in the data for an endoscopic group $H$ for $(\tilde{G}, \alpha)$ and it is this information which allows us to formulate a matching theorem without the assumption (4.3.2) of [Sh6]. Moreover in relating the embeddings ${ }^{L} H \hookrightarrow^{L} \tilde{G}$ relevant to our present problem to the embeddings ${ }^{L} H \hookrightarrow{ }^{L} G$ from $L$-indistinguishability we find a remarkable quasicharacter on $\tilde{H}(\mathbf{R}) \simeq H(\mathbf{C})$ which allows us to dispense with the "cross-section for the norm" in [Sh6] (cf. Lemma 6.4).

As always, the twisted orbital integrals must be normalized. The normalization factors will be written in a form suitable for global applications [L3] and, more specifically, in a form to reflect the connection with $L$-indistinguishability for real groups. The proof of Theorem 7.1 itself relies heavily on the proof of the matching theorem for $L$-indistinguishability (see [Sh5] for an outline of the latter proof).

We will follow the notation of [Sh1]-[Sh7] as closely as possible, especially with respect to $L$-group data. However, we now write $G(\mathbf{C})$ and $G(\mathbf{R})$ in place of $\mathbf{G}$ and $G$. The definitions in this paper may be presented in greater generality (cf. [Sh7]); in the general case there is no such intimate tie with $L$-indistinguishability.

2. The groups $G, \tilde{G}$ and the automorphism $\alpha$. Let $G$ be a connected reductive linear algebraic group defined over R. Assume that $G$ is quasisplit over $\mathbf{R}$. In fixing the usual $L$-group data, we take $G$ itself for $G^{*}$, a quasi-split inner form of $G$, and the identity map for $\psi$, an inner twist from $G$ to $G^{*}$. Then $B^{*}$ will be a Borel subgroup over $\mathbf{R}$ in $G$, and $T^{*}$ a maximal torus over $\mathbf{R}$ in $B^{*}$. We form the dual $\left({ }^{L} G^{0},{ }^{L} B^{0},{ }^{L} T^{0},\left\{X_{r}\right\}\right)$ with $r \in \Sigma\left({ }^{L} B^{0},{ }^{L} T^{0}\right)$, the set of simple roots of ${ }^{L} T^{0}$ in ${ }^{L} B^{0}$. In fact it will be convenient to have fixed a root vector $X_{r}$, for any root $r$ of ${ }^{L} T^{0}$ in ${ }^{L} G^{0}$. We therefore fix a Chevalley basis and take for $\left\{X_{r}, r \in \Sigma\left({ }^{L} B^{0},{ }^{L} T^{0}\right)\right\}$ the vectors so provided. Then ${ }^{L} G={ }^{L} G^{0} \rtimes W$, with $\sigma_{G}$ denoting the action of $1 \times \sigma \in W$ on ${ }^{L} G^{0}$. See [Sh 3, 4, or 5] for further explanation of the notation.

Let $\tilde{G}$ be the group obtained from $G$ by restriction of scalars from $\mathbf{C}$ to $\mathbf{R}$. We realize $\tilde{G}$ as $G \times G$ with Galois automorphism $\sigma_{\tilde{G}}:(x, y) \rightarrow$ $\left(\sigma_{G}(y), \sigma_{G}(x)\right)$. Then $\tilde{B}^{*}=B^{*} \times B^{*}$ will be the distinguished Borel subgroup defined over $\mathbf{R}$ and $\tilde{T}^{*}=T^{*} \times T^{*}$. We realize the $L$-group ${ }^{L} \tilde{G}$ of $\tilde{G}$ as follows. Set ${ }^{L} \tilde{G}^{0}={ }^{L} G^{0} \times{ }^{L} G^{0},{ }^{L} \tilde{B}^{0}={ }^{L} B^{0} \times{ }^{L} B^{0},{ }^{L} \tilde{T}^{0}={ }^{L} T^{0} \times{ }^{L} T^{0}$, $X_{\left(r, r^{\prime}\right)}=\left(X_{r}, X_{r^{\prime}}\right)$ for all roots $r, r^{\prime}$ of ${ }^{L} T^{0}$ in ${ }^{L} G^{0}$, and define $\sigma_{\tilde{G}}$ : ${ }^{L} \tilde{G}^{0} \rightarrow{ }^{L} \tilde{G}^{0}$ by $\sigma_{\tilde{G}}(g, h)=\left(\sigma_{G}(h), \sigma_{G}(g)\right), g, h \in{ }^{L} G^{0}$. Then ${ }^{L} \tilde{G}={ }^{L} \tilde{G}^{0} \rtimes W$, with $\mathbf{C}^{x} \times 1$ acting trivially and $1 \times \sigma$ by $\sigma_{\tilde{G}}$. 
Let $\alpha: \tilde{G} \rightarrow \tilde{G}$ be the automorphism $(x, y) \rightarrow(y, x)$. We take the standard dual automorphism (cf. [Sh7]) of $\alpha$, and denote it by $\alpha$ also. Thus:

$$
\alpha((g, h) \times w)=(h, g) \times w, \quad g, h \in{ }^{L} G^{0}, w \in W .
$$

3. Endoscopic groups for $(\tilde{G}, \alpha)$. The following is a special case of the definitions in [Sh7]. Let $s \in{ }^{L} \tilde{G}^{0}$. Then we set $N(s)=s \alpha(s)$, $\operatorname{Cent}\left(N(s),{ }^{L} \tilde{G}^{0}\right)=\left\{g \in{ }^{L} \tilde{G}^{0}: g^{-1} N(s) g=N(s)\right\}$ and $\operatorname{Cent}_{\alpha}\left(s,{ }^{L} \tilde{G}^{0}\right)=$ $\left\{g \in{ }^{L} \tilde{G}^{0}: g^{-1} s \alpha(g)=s\right\}$. Call $s \alpha$-semisimple if $\operatorname{Cent}_{\alpha}\left(s,{ }^{L} \tilde{G}^{0}\right)$ is reductive. In $\S 4$ we will observe that $s$ is $\alpha$-semisimple if and only if $N(s)$ is semisimple (cf. Lemma 4.2). Let $\tilde{Z}^{W}$ be the group of $W$-invariants in the center of ${ }^{L} \tilde{G}^{0}$. Thus $\tilde{Z}^{W}={ }^{L} \tilde{G}^{0} \cap \operatorname{Center}\left({ }^{L} \tilde{G}\right)=\left\{\left(g, \sigma_{G}(g)\right) \times 1 \times 1: g \in\right.$ Center $\left.\left({ }^{L} G^{0}\right)\right\}$. Also

$$
\operatorname{Cent}_{\alpha}\left(s z,{ }^{L} \tilde{G}^{0}\right)=\operatorname{Cent}_{\alpha}\left(s,{ }^{L} \tilde{G}^{0}\right), \quad s \in{ }^{L} \tilde{G}^{0}, z \in \tilde{Z}^{W} .
$$

We will now use $s$ to denote a coset of $\tilde{Z}^{W}$ in ${ }^{L} \tilde{G}^{0}$ and $\operatorname{Cent}_{\alpha}\left(s,{ }^{L} \tilde{G}^{0}\right)$ to denote $\operatorname{Cent}_{\alpha}\left(a,{ }^{L} \tilde{G}^{0}\right)$ for $a$ in the coset $s$. Following [Sh7], we consider tuples

$$
\left(s,{ }^{L} H_{s}^{0},{ }^{L} B_{s}^{0},{ }^{L} T_{s}^{0},\{Y\}, \rho_{s}\right)
$$

where

(i) $s \in{ }^{L} \tilde{G}^{0}$ is a coset of $\tilde{Z}^{W}$ consisting of $\alpha$-semisimple elements,

(ii) ${ }^{L} H_{s}^{0}=\left(\operatorname{Cent}_{\alpha}\left(s,{ }^{L} \tilde{G}^{0}\right)\right)^{0}$,

(iii) ${ }^{L} B_{s}^{0}$ is a Borel subgroup of ${ }^{L} H_{s}^{0}$,

(iv) ${ }^{L} T_{s}^{0} \subset^{L} B_{s}^{0}$ is a maximal torus in ${ }^{L} H_{s}^{0}$,

(v) $\{Y\}$ is a set of root vectors for the simple roots of ${ }^{L} T_{s}^{0}$ in ${ }^{L} B_{s}^{0}$,

(vi) $\rho_{s}: W \rightarrow \operatorname{Aut}\left({ }^{L} H_{s}^{0},{ }^{L} B_{s}^{0},{ }^{L} T_{s}^{0},\{Y\}\right)$ is a homomorphism which factors through $\operatorname{Gal}(\mathbf{C} / \mathbf{R})$ and is "realized in $\operatorname{Cent}_{\alpha}\left(s,{ }^{L} \tilde{G}\right)$ ", i.e. $\rho_{s}(w)=$ ad $\left.n(w)\right|_{L_{s}}, \quad w \in W$, for some $n(w) \in{ }^{L} \tilde{G}^{0} \times w$ such that $n(w)^{-1} a \alpha(n(w))=a$ for each $a$ in the coset $s$.

Let ${ }^{L} H_{s}={ }^{L} H_{s}^{0} \rtimes W$, the action of $W$ on ${ }^{L} H_{s}^{0}$ being that defined by $\rho_{s}$. Often we will write $\sigma_{s}$ for the automorphism $\rho_{s}(1 \times \sigma)$, and abbreviate $\left(s,{ }^{L} H_{s}^{0}, \ldots, \rho_{s}\right)$ by $\left(s,{ }^{L} H_{s}\right)$.

Two tuples

$$
\left(s,{ }^{L} H_{s}^{0},{ }^{L} B_{s}^{0},{ }^{L} T_{s}^{0},\{Y\}, \rho_{s}\right) \text { and }\left(s^{\prime},{ }^{L} H_{s^{\prime}}^{0},{ }^{L} B_{s^{\prime}}^{0}{ }^{L} T_{s^{\prime}}^{0},\left\{Y^{\prime}\right\}, \rho_{s^{\prime}}\right)
$$

are equivalent if there exists $g \in{ }^{L} \tilde{G}^{0}$ such that ${ }^{L} H_{s^{\prime}}^{0}=g^{-1 L} H_{s}^{0} g,{ }^{L} B_{s^{\prime}}^{0}=$ $g^{-1 L} B_{s}^{0} g, \quad{ }^{L} T_{s^{\prime}}^{0}=g^{-1 L} T_{s}^{0} g, \quad\left\{Y^{\prime}\right\}=\left\{\operatorname{Ad} g^{-1}(Y)\right\} \quad$ and if $n(w) \in$ Cent ${ }_{\alpha}\left(s,{ }^{L} \tilde{G}\right)$ realizes $\rho_{s}(w)$ then $g^{-1} n(w) g$ lies in Cent $\alpha\left(s^{\prime},{ }^{L} \tilde{G}\right)$ and realizes $\rho_{s^{\prime}}(w), w \in W$. The set of all equivalence classes will be denoted 
$\Im(\tilde{G}, \alpha)$. Using the results of the next section and Lemma 2.3.3 of [Sh4], we may show that $\subseteq(\tilde{G}, \alpha)$ is a finite set. Since this fact will not be needed we omit the proof.

Finally, we call a quasi-split group $H$ over $\mathbf{R}$ an endoscopic group for $(\tilde{G}, \alpha)$ if some ${ }^{L} H_{s}$ as above is an $L$-group for $H$.

4. The relation between endoscopic groups for $(\tilde{G}, \alpha)$ and endoscopic groups for $G$. By the endoscopic groups for $G$ we mean the groups " $H$ " of [Sh4], i.e. essentially the groups of [L1]. The set $\subseteq(G)$, or $\subseteq(G, 1)$ in the more general notation of [Sh7], and the tuples used in its definition will be taken from [Sh4] (...there is a small difference in the definitions of [L3]).

We embed ${ }^{L} G$ "diagonally" in ${ }^{L} \tilde{G}$, i.e. by the map $g \times w \rightarrow(g, g) \times w$, $g \in{ }^{L} G^{0}, w \in W$, and will frequently identify ${ }^{L} G$ with its image in ${ }^{L} \tilde{G}$. As in [Sh4], $Z^{W}$ will denote the set of $W$-invariants in the center of ${ }^{L} G^{0}$.

By an $\alpha$-conjugacy class in ${ }^{L} \tilde{G}^{0}$, we will mean a set $\left\{g^{-1} a \alpha(g)\right.$; $\left.g \in{ }^{L} \tilde{G}^{0}\right\}$, where $a \in{ }^{L} \tilde{G}^{0}$.

\section{LEMMA 4.1.}

(i) Each $\alpha$-conjugacy class in ${ }^{L} \tilde{G}^{0}$ contains an element of the form $(x, 1), x \in{ }^{L} G^{0}$.

(ii) For $x \in{ }^{L} G^{0}$, $\operatorname{Cent}_{\alpha}\left((x, 1),{ }^{L} \tilde{G}^{0}\right)=\operatorname{Cent}\left(x,{ }^{L} G^{0}\right)$.

Here, of course, $\operatorname{Cent}\left(x,{ }^{L} G^{0}\right)$ has been identified with its image in ${ }^{L} \tilde{G}$ under the diagonal map.

Proof. Let $a=\left(g_{1}, g_{2}\right) \in{ }^{L} \tilde{G}^{0}, g=\left(1, g_{2}\right)$. Then $g^{-1} a \alpha(g)=$ $\left(1, g_{2}^{-1}\right)\left(g_{1}, g_{2}\right)\left(g_{2}, 1\right)=\left(g_{1} g_{2}, 1\right)$, so that (i) is proved. (ii) is also a simple calculation.

LEMMA 4.2. $a \in{ }^{L} \tilde{G}^{0}$ is $\alpha$-semisimple if and only if $N(a)=a \alpha(a)$ is semisimple.

Proof. Let $a \in{ }^{L} \tilde{G}^{0}$. Choose $g \in{ }^{L} \tilde{G}^{0}$ such that $g^{-1} a \alpha(g)=(x, 1)$, for suitable $x \in{ }^{L} G^{0}$. Then

$$
\operatorname{Cent}_{\alpha}\left(a,{ }^{L} \tilde{G}^{0}\right)=g \operatorname{Cent}_{\alpha}\left((x, 1),{ }^{L} \tilde{G}^{0}\right) g^{-1}=g \operatorname{Cent}\left(x,{ }^{L} G^{0}\right) g^{-1} .
$$

On the other hand, $N(a)=g(x, x) g^{-1}$, so that

$$
\operatorname{Cent}\left(N(a),{ }^{L} \tilde{G}^{0}\right)=g\left(\operatorname{Cent}\left(x,{ }^{L} G^{0}\right) \times \operatorname{Cent}\left(x,{ }^{L} G^{0}\right)\right) g^{-1} .
$$

The lemma then follows from standard facts. 
LEMMA 4.3. Let $s$ be a coset of $\tilde{Z}^{W}$ in ${ }^{L} \tilde{G}^{0}$ consisting of $\alpha$-semisimple elements. Then there exists $g \in{ }^{L} \tilde{G}^{0}$ such that $s^{\prime}=g^{-1} s \alpha(g)$ has the property that $\left\{a \alpha(a): a \in s^{\prime}\right\}$ is contained in ${ }^{L} G^{0}$. Then $\left\{a \alpha(a): a \in s^{\prime}\right\}$ is contained in a unique coset of $Z^{W}$ in ${ }^{L} G^{0}$. This coset, to be denoted $N\left(s^{\prime}\right)$, consists of semisimple elements.

Proof. Let $a \in s$. Choose $g \in{ }^{L} \tilde{G}^{0}$ such that $g^{-1} a \alpha(g)=(x, 1)$, where $x \in{ }^{L} G^{0}$ is semisimple. Let $s^{\prime}=(x, 1) \tilde{Z}^{W}$. Then if $b \in s^{\prime}, b \alpha(b)=$ $(x, x)\left(z \sigma_{G}(z), z \sigma_{G}(z)\right)$, for some $z \in \operatorname{Cent}\left({ }^{L} G^{0}\right)$. Thus, with our identifications, $b \alpha(b) \in x Z^{W}$, a coset of $Z^{W}$ in ${ }^{L} G^{0}$ consisting of semisimple elements. The rest is clear.

LEMMA 4.4. Each element of $\subseteq(\tilde{G}, \alpha)$ has a representative $\left(s,{ }^{L} H_{s}\right)$ such that $\left(N(s),{ }^{L} H_{s}\right)$ is a representative for an element of $\mathfrak{S}(G)$ i.e. such that $\{a \alpha(a): a \in s\}$ is contained in ${ }^{L} G^{0}$ (...so that $N(s)$ is defined $),{ }^{L} H_{s}^{0}$ coincides with $\left(\operatorname{Cent}\left(N(s),{ }^{L} G^{0}\right)\right)$, and $\rho_{s}$ is "realized in $\operatorname{Cent}\left(N(s),{ }^{L} G\right)$. "

Proof. We may take $s=(x, 1) \tilde{Z}^{W}$, some $x \in{ }^{L} T^{0}$. Then $N(s)=x Z^{W}$ and $\operatorname{Cent}_{\alpha}\left(s,{ }^{L} \tilde{G}^{0}\right)=\operatorname{Cent}\left(N(s),{ }^{L} G^{0}\right)$. We may also assume that ${ }^{L} T_{s}^{0}=$ ${ }^{L} T^{0},{ }^{L} B_{s}^{0}={ }^{L} B^{0} \cap{ }^{L} H_{s}^{0}\left(\ldots{ }^{L} T^{0}\right.$ and ${ }^{L} B^{0}$ being identified with their images in $\left.{ }^{L} \tilde{G}^{0}\right)$ and that $\{Y\}=\left\{X_{r}: r \in \Sigma\left({ }^{L} B^{0} \cap{ }^{L} H_{s}^{0},{ }^{L} T^{0}\right)\right\}$. Then $\rho_{s}$ is a homomorphism of $W$ into $\operatorname{Aut}\left({ }^{L} H_{s}^{0},{ }^{L} B^{0} \cap{ }^{L} H_{s}^{0},{ }^{L} T^{0},\{Y\}\right)$. Suppose that $\rho_{s}(w)=$ ad $\left.n(w)\right|_{L_{H_{s}^{0}}}$, where $n(w) \in{ }^{L} \tilde{G}^{0} \times w$ satisfies $n(w)^{-1}(x, 1) \alpha(n(w))=(x, 1)$ (cf. (vi) in §3). Then $n(w)^{-1}(x, x) n(w)=$ $(x, x)$. Also, if $n(w)=\left(n_{1}(w), n_{2}(w)\right) \times w$ then calculation shows that for $w \in \mathbf{C}^{x} \times 1$ we have $n_{1}(w)=n_{2}(w)$ lies in the center of ${ }^{L} H_{s}^{0}$ and for $w=1 \times \sigma$ we have $n_{1}(w)=x n_{2}(w)$. Thus for all $w \in W, \rho_{s}(w)=$ ad $\left.m(w)\right|_{L_{s}}$ where $m(w)=\left(n_{1}(w), n_{1}(w)\right) \times w \in{ }^{L} G$. Also, $m(w)$ centralizes $(x, x)$. Thus $\rho_{s}$ is "realized in $\operatorname{Cent}\left(N(s),{ }^{L} G\right)$ " and the lemma is proved.

LEMMA 4.5. The correspondence in Lemma 4.4 induces a map

$$
\Re: \Im(\tilde{G}, \alpha) \rightarrow \Im(G) .
$$

Proof. We have to show that if $\left(s,{ }^{L} H_{s}\right)$ and $\left(s^{\prime},{ }^{L} H_{s^{\prime}}\right)$ are as in Lemma 4.4 , representing the same element of $\subseteq(\tilde{G}, \alpha)$, then the 5-tuples defining ${ }^{L} H_{s}$ and ${ }^{L} H_{s^{\prime}}$ are conjugate under ${ }^{L} G^{0}$. They are conjugate under ${ }^{L} \tilde{G}^{0}$, by definition. It is easily checked that this conjugation may be replaced by one from ${ }^{L} G^{0}$. 
The map $\Re$ need not be injective, as the example that $G$ is a compact torus shows. However $\Re$ does have finite fibers (which implies that $\Im(\tilde{G}, \alpha)$ is finite, as asserted in the last section). Reversing the construction in the proof of Lemma 4.4 shows that $\mathcal{R}$ is surjective.

5. Allowed embeddings of ${ }^{L} H_{s}$ in ${ }^{L} \tilde{G}$. Fix an element of $\subseteq(\tilde{G}, \alpha)$, with representative $\left(s,{ }^{L} H_{s}\right)$ chosen as in the proof of Lemma 4.4. In particular, $s=(x, 1) \tilde{Z}^{W}, x \in{ }^{L} T^{0}$, and ${ }^{L} H_{s}^{0}=\left(\operatorname{Cent}_{\alpha}\left(s,{ }^{L} \tilde{G}^{0}\right)\right)^{0}=$ $\left(\operatorname{Cent}\left(N(s),{ }^{L} G^{0}\right)\right)^{0}$. We may further assume that ${ }^{L} H_{s}^{0}$ is in standard position (cf. [Sh3, §2.2, Ex. 4.3.1]).

Suppose that $\xi:{ }^{L} H_{s} \hookrightarrow{ }^{L} G$ is an admissible embedding, as in $L$-indistinguishability [L1], [Sh3]. Here we regard ${ }^{L} H_{s}^{0}$ as a subgroup of ${ }^{L} G^{0}$ yet to be embedded diagonally in ${ }^{L} \tilde{G}^{0}$, and assume that $\left.\xi\right|_{L} H_{s}^{0}$ is the inclusion map. The "diagonal" embedding of ${ }^{L} G$ in ${ }^{L} \tilde{G}$ then yields an embedding of ${ }^{L} H_{s}$ in ${ }^{L} \tilde{G}$, again denoted $\xi$. Explicitly, $\xi$ is of the form:

$$
\begin{aligned}
& \xi(h \times 1 \times 1)=(h, h) \times 1 \times 1, \quad h \in{ }^{L} H_{s}^{0}, \\
& \xi(1 \times z \times 1)=\left(\xi_{0}(z), \xi_{0}(z)\right) \times z \times 1, \quad z \in \mathbf{C}^{x},
\end{aligned}
$$

where $\xi_{0}: \mathbf{C}^{x} \rightarrow \operatorname{Cent}\left({ }^{L} H_{s}^{0}\right)$ is a homomorphism satisfying $\xi_{0}(\bar{z})=$ $\boldsymbol{\sigma}_{s}\left(\xi_{0}(z)\right), z \in \mathbf{C}^{x}$, and

$$
\xi(1 \times 1 \times \sigma)=\left(n_{0}, n_{0}\right) \times 1 \times \sigma,
$$

where $n_{0} \in{ }^{L} G^{0}$ normalizes ${ }^{L} T^{0}, n_{0} \sigma_{G}\left(n_{0}\right)=\xi_{0}(-1)$ and $n_{0} \times 1 \times \sigma \in{ }^{L} G$ acts on ${ }^{L} H_{s}^{0}$ as $\sigma_{s}=\rho_{s}(1 \times \sigma)$. It follows immediately that $\xi\left({ }^{L} H_{s}\right) \subset$ $\operatorname{Cent}\left(N(s),{ }^{L} G\right)$. However, our present problem dictates (cf. §8) that we consider embeddings for which the image of ${ }^{L} H_{s}$ is contained in $\operatorname{Cent}_{\alpha}\left(s,{ }^{L} \tilde{G}\right)$. That this is a quite different condition is indicated even by the example that $G$ is a compact torus.

Definition 5.1. Let $\left(s,{ }^{L} H_{s}\right)$ be a representative for an element of $\subseteq(\tilde{G}, \alpha)$. Then $\tilde{\xi}:{ }^{L} H_{s} \hookrightarrow{ }^{L} \tilde{G}$ is an allowed embedding if:

(i) $\tilde{\xi}$ is an admissible homomorphism, i.e. $\tilde{\xi}$ is a homomorphism such that $\tilde{\xi}\left({ }^{L} H_{s}^{0} \times w\right) \subset{ }^{L} \tilde{G}^{0} \times w, w \in W$,

(ii) on ${ }^{L} H_{s}^{0}, \tilde{\xi}$ is the inclusion mapping, and

(iii) $\tilde{\xi}\left({ }^{L} H_{s}\right) \subset \operatorname{Cent}_{\alpha}\left(s,{ }^{L} \tilde{G}\right)$.

We return to our choice $s=(x, 1) \tilde{Z}^{W}$, etc. Once again it is more convenient to regard ${ }^{L} H_{s}^{0}$ as a subgroup of ${ }^{L} G^{0}$ yet to be embedded 
diagonally in ${ }^{L} \tilde{G}^{0}$. Then an allowed embedding $\tilde{\xi}:{ }^{L} H_{s} \hookrightarrow{ }^{L} \tilde{G}$ is of the form:

$$
\begin{aligned}
& \tilde{\xi}(h \times 1 \times 1)=(h, h) \times 1 \times 1, \quad h \in{ }^{L} H_{s}^{0}, \\
& \tilde{\xi}(1 \times z \times 1)=\left(\tilde{\xi}_{0}(z), \tilde{\xi}_{0}(z)\right) \times z \times 1, \quad z \in \mathbf{C}^{x},
\end{aligned}
$$

where $\tilde{\xi}_{0}$ satisfies the same conditions as $\xi_{0}$ earlier, and

$$
\tilde{\xi}(1 \times 1 \times \sigma)=\left(x m_{0}, m_{0}\right) \times 1 \times \sigma
$$

where $m_{0} \in{ }^{L} G^{0}$ normalizes ${ }^{L} T^{0}, x m_{0} \sigma_{G}\left(m_{0}\right)=\tilde{\xi}_{0}(-1)$, and $m_{0} \times 1 \times \sigma$ $\epsilon^{L} G$ acts on ${ }^{L} H_{s}^{0}$ as $\sigma_{s}\left(\ldots\right.$ then also $x m_{0} \times 1 \times \sigma$ acts on ${ }^{L} H_{s}^{0}$ as $\sigma_{s}$, as we have already used in the proof of Lemma 4.2).

Let ${ }^{L} \tilde{H}_{s}^{0}={ }^{L} H_{s}^{0} \times{ }^{L} H_{s}^{0}$. We of course regard ${ }^{L} \tilde{H}_{s}^{0}$ as a subgroup of ${ }^{L} \tilde{G}^{0}$. Define an action of $W$ on ${ }^{L} \tilde{H}_{s}^{0}$ by requiring $\mathbf{C}^{x} \times 1$ to act trivially and $1 \times \sigma$ to act by the automorphism $\left(h_{1}, h_{2}\right) \rightarrow\left(\sigma_{s}\left(h_{2}\right), \sigma_{s}\left(h_{1}\right)\right)$. If ${ }^{L} H_{s}$ is the $L$-group of $H$ then ${ }^{L} \tilde{H}_{s}$ is the $L$-group of $\tilde{H}=\operatorname{Res}_{\mathrm{R}}^{\mathrm{C}} H$.

LEMMA 5.2. Let $\tilde{\xi}$ be an allowed embedding of ${ }^{L} H_{s}$ in ${ }^{L} \tilde{G}$ and $\xi$ be an admissible embedding of ${ }^{L} H_{s}$ in ${ }^{L} G \subset{ }^{L} \tilde{G}$. Then

$$
\tilde{\xi}(h \times w)=a(w) \xi(h \times w), \quad h \in{ }^{L} H_{s}^{0}, w \in W,
$$

where $a(w)$ is a 1-cocycle of $W$ in $\operatorname{Cent}\left({ }^{L} \tilde{H}_{s}^{0}\right)$.

Proof. This follows easily from our explicit description of $\tilde{\xi}$ and $\xi$. The details are omitted.

Suppose that $\tilde{\xi}, \tilde{\xi}^{\prime}$ are both allowed embeddings of ${ }^{L} H_{s}$ in ${ }^{L} \tilde{G}$. Then $\tilde{\xi}^{\prime}(w)=b(w) \tilde{\xi}(w), w \in W$, where $w \rightarrow b(w)$ is a 1 -cocycle of $W$ in the center of ${ }^{L} H_{s}^{0}$ embedded diagonally in ${ }^{L} \tilde{G}^{0}$. We conclude then that the image of ${ }^{L} H_{s}$ under an allowed embedding is independent of the choice of embedding; we write thus simply "Image ${ }^{L} \boldsymbol{H}_{s}$." Suppose next that $\left(s,{ }^{L} \boldsymbol{H}_{s}\right)$ and $\left(s^{\prime},{ }^{L} H_{s^{\prime}}\right)$ are equivalent in the sense of $\S 3$. Fix $g \in{ }^{L} \tilde{G}^{0}$ as in the definition. Suppose that $\tilde{\xi}$ is an allowed embedding of ${ }^{L} H_{s}$ in ${ }^{L} \tilde{G}$. Then ad $g$ and $\tilde{\xi}$ determine an allowed embedding of ${ }^{L} H_{s^{\prime}}$ in ${ }^{L} \tilde{G}$. We conclude then that there is an allowed embedding of ${ }^{L} H_{s}$ in ${ }^{L} \tilde{G}$ if and only if there is such an embedding of ${ }^{L} H_{s^{\prime}}$. Moreover, when embeddings exist we have $g^{-1}\left(\right.$ Image $\left.^{L} H_{s}\right) g=\operatorname{Image}^{L} H_{s^{\prime}}$.

We defer a study of the existence of allowed embeddings. Recall, however, that if the center of ${ }^{L} G^{0}$ is connected then ${ }^{L} H_{s}$ embeds admissibly in ${ }^{L} G[\mathbf{L 1}]$. The proof of this result can be used to show also that there is an allowed embedding of ${ }^{L} H_{s}$ in ${ }^{L} \tilde{G}$.

6. Ingredients for the matching theorem. Fix an element of $\subseteq(\tilde{G}, \alpha)$ with representative $\left(s,{ }^{L} H_{s}\right)$ satisfying $s=(x, 1) \tilde{Z}^{W}$, etc., as in the last section. We assume that $\tilde{\xi}:{ }^{L} H_{s} \hookrightarrow{ }^{L} \tilde{G}$ is an allowed embedding. The main 
purpose of this section is to attach to $\tilde{\xi}$ normalizing factors to appear in the matching theorem of the next section. We will assume also that there is an admissible embedding of ${ }^{L} H_{s}$ in ${ }^{L} G$, say $\xi$. The choice of $\xi$ will not affect the normalization factors (cf. Lemma 6.2), but we write individual terms in the factors in a way that involves $\xi$, in order to make clear the relation with the factors from $L$-indistinguishability.

Let $H$ be an endoscopic group for $(\tilde{G}, \alpha)$ with $L$-group ${ }^{L} H_{s}$. We fix a Borel subgroup $B_{H}$ over $\mathbf{R}$ containing the maximal torus $T_{H}$ over $\mathbf{R}$, and assume that $X^{*}\left(T_{H}\right)=X_{*}\left({ }^{L} T^{0}\right)=X^{*}\left(T^{*}\right)$ and that $\Sigma\left(B_{H}, T_{H}\right)$ is the dual of $\Sigma\left({ }^{L} B_{s}^{0},{ }^{L} T^{0}\right)$. The group $\tilde{H}=\operatorname{Res}_{\mathbf{R}}^{\mathbf{C}} H$ will also play a role. We set $\tilde{B}_{H}=B_{H} \times B_{H}$ and $\tilde{T}_{H}=T_{H} \times T_{H} ;{ }^{L} \tilde{H}_{s}$, which appeared in the last section, is an $L$-group for $\tilde{H}$.

Since $H$ is also an endoscopic group for $G$ we may invoke many of the definitions from $L$-indistinguishability (cf. [L1], [Sh4]). Let $T$ be a maximal torus over $\mathbf{R}$ in $G$. A pseudodiagonalization (p.d.) $\eta$ of $T$ is a map from $T$ to $T^{*}$ of the form $T \stackrel{\text { ad } x}{\rightarrow} T_{0} \stackrel{\text { ad } m}{\rightarrow} T^{*}$, where $x \in \mathfrak{A}(T)$ [L1], $T_{0}=$ $x T x^{-1}$ is standard (i.e. the maximal $R$-split torus in $T_{0}$ lies in $T^{*}$ ) and $m$ belongs to the Levi group attached to $T_{0}$. Then $\sigma_{(T, \eta)}$ denotes the transfer, by $\eta$, of the Galois action on $T$ to $T^{*}$, and to $X^{*}\left(T^{*}\right)=X_{*}\left({ }^{L} T^{0}\right)$, $X_{*}\left(T^{*}\right)=X^{*}\left({ }^{L} T^{0}\right)$ and ${ }^{L} T^{0}=X_{*}\left({ }^{L} T^{0}\right) \otimes \mathbf{C}^{x}$.

The set $\mathcal{T}_{H}(G)=\left\{(T, \eta): \sigma_{(T, \eta)} \in \Omega\left({ }^{L} H_{s}^{0},{ }^{L} T^{0}\right) \sigma_{s}\right\}$, where $\Omega\left({ }^{L} H_{s}^{0},{ }^{L} T^{0}\right)$ denotes the Weyl group of $\left({ }^{L} H_{s}^{0},{ }^{L} T^{0}\right)$, is the starting point for the definitions of [Sh4, §2.4]. We will use it again. First, because $G$ is quasi-split over $\mathbf{R}$, for each maximal torus $T^{\prime}$ over $\mathbf{R}$ in $H$ there exists $h \in H(\mathbf{C})$ and $(T, \eta) \in \mathcal{T}_{H}(G)$ such that $h T^{\prime} h^{-1}=T_{H}$ and

$$
X^{*}\left(T^{\prime}\right) \stackrel{\text { ad } h}{\rightarrow} X^{*}\left(T_{H}\right)=X^{*}\left(T^{*}\right) \stackrel{\eta^{-1}}{\rightarrow} X^{*}(T)
$$

lifts to an isomorphism $i(h, \eta): T^{\prime} \rightarrow T$ over $\mathbf{R}$. We say that $\gamma^{\prime} \in H(\mathbf{R})$ originates from $\gamma \in G(\mathbf{R})$ via $(T, \eta)$ if $\gamma^{\prime}$ is the preimage of $\gamma$ under some such map $i(h, \eta)$.

Recall that $s=(x, 1) \tilde{Z}^{W}$. Any element of this coset is of the form $a=\left(x z, \sigma_{G}(z)\right)$, where $z$ is in the center of ${ }^{L} G^{0}$. But $a \alpha(a)=$ $\left(x z \sigma_{G}(z), x z \sigma_{G}(z)\right)$, an element of ${ }^{L} T^{0}=\operatorname{Hom}\left(X^{*}\left({ }^{L} T^{0}\right), \mathbf{C}^{x}\right)$. Also $\sigma_{s}(x)$ $=x$. Thus $\{a \alpha(a): a \in s\}$ defines a family of quasicharacters on $X^{*}\left({ }^{L} T^{0}\right)$, each invariant under $\sigma_{(T, \eta)}$, for any $(T, \eta) \in \widetilde{T}_{H}(G)$. Fix $(T, \eta) \in \mathcal{T}_{H}(G)$. Then, on transfer to $T$ via $\eta$, we get a family of quasicharacters on $X_{*}(T)$, each invariant under $\sigma_{T}$. On $X_{*}\left(T_{\mathrm{sc}}\right)$, the span of the coroots of $T$ in $G$, these quasicharacters all coincide and so we have defined a single quasicharacter of the type used in $L$-indistinguishability (cf. [L1], also [Sh4, §2.4]). Moreover on $\left\{\lambda^{\vee} \in X_{*}(T): \sigma_{T} \lambda^{\vee}=-\lambda^{\vee}\right\}$, the quasicharacters 
coincide again. We therefore obtain a single character on

$$
\left\{\lambda^{\vee} \in X_{*}(T): \sigma_{T} \lambda^{\vee}=-\lambda^{\vee}\right\} /\left\{\mu^{\vee}-\sigma_{T} \mu^{\vee}: \mu^{\vee} \in X_{*}(T)\right\}
$$

and thence by Tate-Nakayama duality, a character on $H^{1}(T)=$ $H^{1}(\mathrm{Gal}(\mathbf{C} / \mathbf{R}), T(\mathbf{C}))$. Unless otherwise indicated, $\kappa$ will denote both the quasicharacter on $X_{*}\left(T_{\mathrm{sc}}\right)$ and the character on $H^{1}(T)$ attached to $s$ and the $\operatorname{pair}(T, \eta) \in \mathcal{T}_{H}(G)$.

With $G$ embedded diagonally in $\tilde{G}$, we have $\tilde{T}=\operatorname{Res}_{\mathbf{R}}^{\mathbf{C}} T$ naturally embedded in $\tilde{G}$ as $\operatorname{Cent}(T, \tilde{G})=T \times T$, for any maximal torus $T$ over $\mathbf{R}$ in $G$. The norm from $\tilde{T}$ to $T$ is obtained from the map $\tilde{T}(\mathbf{R}) \rightarrow T(\mathbf{R})$ defined by $\delta=\left(t, \sigma_{G}(t)\right) \rightarrow \delta \alpha(\delta)=\left(t \sigma_{G}(t), t \sigma_{G}(t)\right)$. As in [Sh6] we regard the norm from $\tilde{G}$ to $G$ (...or from $\tilde{T}$ to $T$ ) as an (injective) map from the set of stable regular $\alpha$-semisimple twisted conjugacy classes in $\tilde{G}(\mathbf{R})$ (...or in $\tilde{T}(\mathbf{R})$ ) to the set of stable regular semisimple conjugacy classes in $G(\mathbf{R})$ (...or to $T(\mathbf{R})$ ). by Lemma 2.4.3(ii) of [Sh6] this norm from $\tilde{G}$ to $G$ can be recovered from the norms from $\tilde{T}$ to $T$, as $T$ ranges over the maximal tori over $\mathbf{R}$ in $G$.

Note that if $\eta: T \rightarrow T^{*}$ is a p.d., then so is $\eta \times \eta: \tilde{T} \rightarrow \tilde{T}^{*}$. Thus we can use $\eta$ to transfer data from $\tilde{T}$ to $\tilde{T}^{*}$ or from $\tilde{T}^{*}$ to $\tilde{T}$.

We come then to the normalizing factors. The admissible embedding $\xi:{ }^{L} H_{s} \hookrightarrow{ }^{L} G$ has been fixed, and ${ }^{L} H_{s}$ chosen to satisfy the conditions of [Sh3, Sh4]. We may therefore write $\xi=\xi\left(\mu^{*}, \lambda^{*}\right)$, for suitable $\mu^{*}, \lambda^{*} \in$ $X_{*}\left({ }^{L} T^{0}\right) \otimes \mathbf{C}$, and define the attached correction (quasi) characters $\Lambda_{(T, \eta)}$ on $T(\mathbf{R})$, for $(T, \eta) \in \mathcal{T}_{H}(G)$. Although the notation does not reflect it, $\Lambda_{(T, \eta)}$ depends on the choice of $\xi$.

Since $\tilde{\xi}:{ }^{L} H_{s} \hookrightarrow{ }^{L} \tilde{G}$ has also been fixed, we have the 1-cocycle $a(w)$ of $W$ in Center $\left({ }^{L} \tilde{H}_{s}^{0}\right)$ from Lemma 5.2. A procedure in [L2] attaches to $a(w)$ a quasicharacter on $\tilde{H}(\mathbf{R})$. This quasicharacter determines a pair $\left(\tilde{\mu}_{0}, \tilde{\lambda}_{0}\right)$ of elements from $X^{*}\left(\tilde{T}_{H}\right) \otimes \mathbf{C}=X_{*}\left({ }^{L} \tilde{T}^{0}\right) \otimes \mathbf{C}$. We may also recover $\left(\tilde{\mu}_{0}, \tilde{\lambda}_{0}\right)$ directly from the 1-cocycle $a(w)$. Thus define $\tilde{\mu}_{0}, \tilde{\lambda}_{0}$ by

$$
\begin{aligned}
& \lambda^{\vee}(a(z \times 1))=z^{\left\langle\tilde{\mu}_{0}, \lambda^{\vee}\right\rangle} \bar{z}^{\left\langle\sigma_{s} \tilde{\mu}_{0}, \lambda^{\vee}\right\rangle}, \quad z \in \mathbf{C}^{x}, \\
& \lambda^{\vee}(a(1 \times \boldsymbol{\sigma}))=e^{2 \pi i\left\langle\tilde{\lambda}_{0}, \lambda^{\vee}\right\rangle}
\end{aligned}
$$

for $\lambda^{\vee} \in X^{*}\left({ }^{L} \tilde{T}^{0}\right)$. Then $\tilde{\mu}_{0}$ is uniquely determined and $\tilde{\lambda}_{0}$ is uniquely determined modulo

$$
X_{*}\left({ }^{L} \tilde{T}^{0}\right)+\left\{\tilde{\lambda}-\tilde{\sigma}_{s} \tilde{\lambda}: \tilde{\lambda} \in X_{*}\left({ }^{L} \tilde{T}^{0}\right) \otimes \mathbf{C}\right\} .
$$

Also

$\tilde{\mu}_{0}-\tilde{\sigma}_{s} \tilde{\mu}_{0} \in X_{*}\left({ }^{L} \tilde{T}^{0}\right), \quad 1 / 2\left(\tilde{\mu}_{0}-\tilde{\sigma}_{s} \tilde{\mu}_{0}\right) \equiv \tilde{\lambda}_{0}+\tilde{\sigma}_{s} \tilde{\lambda}_{0} \bmod X_{*}\left({ }^{L} \tilde{T}^{0}\right)$, 
and

$$
\left\langle\tilde{\mu}_{0}, \lambda^{\vee}\right\rangle=0, \quad\left\langle\tilde{\lambda}_{0}, \lambda^{\vee}\right\rangle \in \mathbf{Z}
$$

whenever $\lambda^{\vee}$ lies in the span of the roots of ${ }^{L} \tilde{T}^{0}$ in ${ }^{L} \tilde{H}_{s}^{0}$ (cf. $\$ 9.1$ of [Sh3]). Here we have used $\tilde{\sigma}_{s}$ to denote the action of $1 \times 1 \times \sigma \in{ }^{L} \tilde{H}_{s}$.

Let $(T, \eta) \in \mathcal{F}_{H}(G)$. Then on transferring $\left(\tilde{\mu}_{0}, \tilde{\lambda}_{0}\right)$ to $\tilde{T}$ using $\eta$ we obtain the data also denoted $\left(\tilde{\mu}_{0}, \tilde{\lambda}_{0}\right)$ for a quasicharacter on $\tilde{T}(\mathbf{R})$ (cf. [Sh3, §4.1]). This quasicharacter will be denoted $a_{(T, \eta)}$.

\section{LEMMA 6.1.}

$$
a_{(T, \eta)} \text { is } \alpha \text {-invariant. }
$$

Proof. We describe $a_{(T, \eta)}$ explicitly. Let $\delta=\left(t, \sigma_{T}(t)\right) \in \tilde{T}(\mathbf{R})$. Write $t$ as $\exp X, X \in \operatorname{Lie}(T(\mathbf{C}))=X_{*}(T) \otimes \mathbf{C}$. Then $\sigma_{T}(t)=\exp \sigma_{T}(\bar{X})$, where if $X=\sum_{i=1}^{n} \lambda_{l}^{\vee} \otimes z_{i}$ then $\sigma_{T}(\bar{X})=\sum_{i=1}^{n} \sigma_{T}\left(\lambda_{l}^{\vee}\right) \otimes \bar{z}_{i}$. Because $a\left(\mathbf{C}^{x} \times 1\right)$ lies in the diagonal subgroup of $\operatorname{Center}\left({ }^{L} \tilde{H}_{s}^{0}\right)$, as is evident from the form of the embeddings $\xi$ and $\tilde{\xi}$ (cf. last section), we must have $\tilde{\mu}_{0}$ lying in the diagonal subspace of $X_{*}\left({ }^{L} \tilde{T}^{0}\right) \otimes \mathbf{C}=\left(X_{*}\left({ }^{L} T^{0}\right) \otimes \mathbf{C}\right) \times\left(X_{*}\left({ }^{L} T^{0}\right) \otimes \mathbf{C}\right)$. Thus we write $\tilde{\mu}_{0}$ as $\left(\mu_{0}, \mu_{0}\right), \mu_{0} \in X_{*}\left({ }^{L} T^{0}\right) \otimes \mathbf{C}$. As usual, we transfer $\mu_{0}$ to $X^{*}(T) \otimes \mathrm{C}$ via $\eta$ without change in notation. Then

$$
a_{(T, \eta)}(\delta)=e^{\mu_{0}\left(X+\sigma_{T}(\bar{X})\right)} .
$$

Since $\alpha(\delta)=\left(\exp \sigma_{T}(\bar{X}), \exp X\right)$ it is now clear that $a_{(T, \eta)}(\alpha(\delta))=$ $a_{(T, \eta)}(\delta)$, and the lemma is proved.

Note that $a_{(T, \eta)}$ is uniquely determined by the class of $a(w)$ in $H^{1}\left(W\right.$, Center $\left.\left({ }^{L} \tilde{H}_{s}^{0}\right)\right)$, but is affected by a change in $\xi$ or $\tilde{\xi}$. The dependence on $\tilde{\xi}$ of our normalization factors is to be expected; the dependence on $\xi$ is not.

Lemma 6.2. Fix $(T, \eta) \in \mathcal{T}_{H}(G)$ and $\delta \in \tilde{T}(\mathbf{R})$. Then $\alpha_{(T, \eta)}(\delta) \Lambda_{(T, \eta)}(\delta \alpha(\delta))$ depends on $\tilde{\xi}$ alone.

Proof. The embedding $\xi$ may be replaced only by $h \times w \rightarrow$ $a_{0}(w) \xi(h \times w)$, where $a_{0}(w)$ is a 1-cocycle of $W$ in the center of ${ }^{L} H_{s}^{0}$ embedded diagonally in the center of ${ }^{L} \tilde{H}_{s}^{0}$. Then $a(w)$ is replaced by $a_{0}(w)^{-1} a(w)$. The cocycle $a_{0}(w)$ defines first a quasicharacter $\chi$ on $H(\mathbf{R})$ and second a quasicharacter $\tilde{\chi}$ on $\tilde{H}(\mathbf{R})$. As before, we use $\eta$ to transfer data and define quasicharacters $\chi_{(T, \eta)}$ on $T(\mathbf{R})$ and $\tilde{\chi}_{(T, \eta)}$ on $\tilde{T}(\mathbf{R})$. Since $\Lambda_{(T, \eta)}$ is replaced by $\chi_{(T, \eta)} \Lambda_{(T, \eta)}$ and $a_{(T, \eta)}$ by $\tilde{\chi}_{(T, \eta)}^{-1} a_{(T, \eta)}$, we have only to show that $\tilde{\chi}_{(T, \eta)}(\delta)=\chi_{(T, \eta)}(\delta \alpha(\delta))$. Define parameters $\mu_{1}, \lambda_{1} \in X_{*}\left({ }^{L} T^{0}\right)$ $\otimes \mathbf{C}$ for $\chi$ as usual; use the same symbols for their transfer to $X^{*}(T) \otimes \mathbf{C}$ 
via $\eta$. For $\tilde{\chi}$ we can use parameters $\tilde{\mu}_{1}=\left(\mu_{1}, \mu_{1}\right), \tilde{\lambda}_{1}=\left(\lambda_{1}, \lambda_{1}\right)$ in $X_{*}\left({ }^{L} \tilde{T}^{0}\right) \otimes \mathbf{C}\left(\ldots\right.$ or $X^{*}(\tilde{T}) \otimes \mathbf{C}$, after transfer $)$. Since $\tilde{\chi}$ is clearly $\alpha$-invariant (see the last proof), we may take $\delta=(\exp X, \exp X), X \in$ $\operatorname{Lie}(T(\mathbf{R}))$. Then $\tilde{\chi}(\delta)=e^{\left\langle 2 \mu_{1}, X\right\rangle}$ and $\chi(\delta \alpha(\delta))=\chi\left(\delta^{2}\right)=e^{\left\langle\mu_{1}, 2 X\right\rangle}$, so that the lemma is proved.

The next lemma is simple but very useful (cf. proof of Lemma 6.4). Each element of $H^{1}(T)$ can be represented by a cocycle $\sigma \rightarrow \exp i \pi \lambda^{\vee}$, where $\lambda^{\vee} \in X_{*}(T)$ and $\sigma_{T} \lambda^{\vee}=-\lambda^{\vee}$. We will use $\exp i \pi \lambda^{\vee}$ to denote this cocycle and its class in $H^{1}(T)$; of course, exp $i \pi \lambda^{\vee}$ also denotes an element of $T(\mathbf{R}) \subset \tilde{T}(\mathbf{R})$. Recall that to $(T, \eta) \in \mathcal{T}_{H}(G)$ and our fundamental datum $s=(x, 1) \tilde{Z}^{W}$ we have attached a character $\kappa$ on $H^{1}(T)$.

LEMMA 6.3.

$$
a_{(T, \eta)}\left(\exp i \pi \lambda^{\vee}\right)=\kappa\left(\exp i \pi \lambda^{\vee}\right)
$$

for all $\lambda^{\vee} \in X_{*}(T)$ such that $\sigma_{T} \lambda^{\vee}=-\lambda^{\vee}$.

Note that the left side alone appears to depend on the choice of $\xi$ and $\tilde{\xi}$. However a quasicharacter $\tilde{\chi}$ as in the last proof annihilates $\exp i \pi \lambda^{\vee}$, if $\lambda^{\vee} \in X_{*}(T)$ and $\sigma_{T} \lambda^{\vee}=-\lambda^{\vee}$. Indeed we then have $i \pi \lambda^{\vee} \in \operatorname{Lie}(T(\mathbf{R}))$, so that $\tilde{\chi}\left(\exp i \pi \lambda^{\vee}\right)=e^{2 \pi l\left\langle\mu_{1}, \lambda^{\vee}\right\rangle}=1$, since $\frac{1}{2}\left(\mu_{1}-\sigma_{T} \mu_{1}\right) \equiv\left(\lambda_{1}+\sigma_{T} \lambda_{1}\right)$ $\bmod X^{*}(T)$ implies that $\left\langle\frac{1}{2}\left(\mu_{1}-\sigma_{T} \mu_{1}\right), \lambda^{\vee}\right\rangle=\left\langle\mu_{1}, \lambda^{\vee}\right\rangle$ lies in Z. It then follows that neither side of the formula depends on $\xi$ or $\tilde{\xi}$.

Proof of Lemma 6.3. First we evaluate the right side. The cocycle $\sigma \rightarrow \exp i \pi \lambda^{\vee}$ corresponds under the Tate-Nakayama isomorphism to the coset of $\lambda^{\vee}$ in

$$
\begin{aligned}
& H^{-1}\left(X_{*}(T)\right) \\
& \quad=\left\{\mu^{\vee} \in X_{*}(T): \sigma_{T} \mu^{\vee}=-\mu^{\vee}\right\} /\left\{\nu^{\vee}-\sigma_{T} \nu^{\vee}: \nu^{\vee} \in X_{*}(T)\right\} .
\end{aligned}
$$

Thus $\kappa\left(\exp i \pi \lambda^{\vee}\right)=\lambda^{\vee}(x)$, where $s=(x, 1) \tilde{Z}^{W}$ was used to define $\kappa$. Note that we have transferred $\lambda^{\vee}$ to ${ }^{L} T^{0}$ via $\eta$.

For the left side, we write $a(z \times 1)=\left(a_{0}(z), a_{0}(z)\right), z \in \mathbf{C}^{x}$, and $a(1 \times \sigma)=\left(x b_{0}, b_{0}\right)$, where $a_{0}(z), b_{0}$ lie in the center of ${ }^{L} H_{s}^{0}$. Since $i \pi \lambda^{\vee} \in \operatorname{Lie}(T(\mathbf{R}))$, we have $a_{(T, \eta)}\left(\exp i \pi \lambda^{\vee}\right)=e^{2 \pi i\left\langle\mu_{0}, \lambda^{\vee}\right\rangle}=\lambda^{\vee}\left(a_{0}(-1)\right)$, where again we have transferred $\lambda^{\vee}$ to ${ }^{L} T^{0}$ without change in notation (cf. proof of Lemma 6.1). On the other hand, $a(1 \times \sigma) \tilde{\sigma}_{s}(a(1 \times \sigma))=a(-1)$ implies that $a_{0}(-1)=x b_{0} \sigma_{s}\left(b_{0}\right)=x b_{0} \sigma_{(T, \eta)}\left(b_{0}\right)$. Since $\sigma_{(T, \eta)} \lambda^{\vee}=-\lambda^{\vee}$, we have that $\lambda^{\vee}\left(a_{0}(-1)\right)=\lambda^{\vee}(x)$, and the lemma is proved. 
We continue with $(T, \eta) \in \mathcal{T}_{H}(G)$ and associated character $\kappa$ on $H^{1}(T)$. Fix a set $\left\{u=\exp i \pi \lambda^{\vee}: \lambda^{\vee} \in X_{*}(T), \sigma_{T} \lambda^{\vee}=-\lambda^{\vee}\right\}$ such that the cocycles $\sigma \rightarrow \exp i \pi \lambda^{\vee}$ form a complete set of (noncohomologous) representatives for the elements of $H^{1}(T)$.

For $f \in C_{c}^{\infty}(\tilde{G}(\mathbf{R}))$, and Haar measures $d t$ on $T(\mathbf{R}), d \tilde{g}$ on $\tilde{G}(\mathbf{R})$ form (cf. [Sh6]):

$$
\Phi_{f}^{(T, \alpha, \kappa)}(\delta, d t, d \tilde{g})=\sum_{u} \kappa(u) \int_{\tilde{G}(\mathbf{R}) / T(\mathbf{R})} f\left(\alpha(\tilde{g}) u \delta \tilde{g}^{-1}\right) \frac{d \tilde{g}}{d t},
$$

for $\delta \in \tilde{T}(\mathbf{R})$ such that $\delta \alpha(\delta)$ is regular. Note that for all $\delta \in \tilde{T}(\mathbf{R}), \delta \alpha(\delta)$ lies in $T(\mathbf{R})^{0}$, the identity component of $T(\mathbf{R})$.

LEMMA 6.4.

$$
\gamma \rightarrow a_{(T, \eta)}(\delta) \Phi_{f}^{(T, \alpha, \kappa)}(\delta, d t, d \tilde{g}),
$$

if $\delta \alpha(\delta)=\gamma, \gamma \in T(\mathbf{R})_{\mathrm{reg}}^{0}=T(\mathbf{R})^{0} \cap G_{\mathrm{reg}}$, is a well-defined function on $T(\mathbf{R})_{\text {reg }}^{0}$.

Proof. By Lemma 6.3,

$$
a_{(T, \eta)}(\delta) \Phi_{f}^{(T, \alpha, \kappa)}(\delta, d t, d \tilde{g})=\sum_{u} a_{(T, \eta)}(u \delta) \int_{\tilde{G}(\mathbf{R}) / T(\mathbf{R})} f\left(\alpha(\tilde{g}) u \delta \tilde{g}^{-1}\right) \frac{d \tilde{g}}{d t}
$$

which we will write as $\Phi(\delta)$. If $\delta \alpha(\delta)=\delta^{\prime} \alpha\left(\delta^{\prime}\right)$ then $\delta^{\prime}=v \delta$, where $v \alpha(v)=1, v \in \tilde{T}(\mathbf{R})$. Then it is easily seen that $v=t^{-1} \alpha(t) u$ for some $t \in \tilde{T}(\mathbf{R})$ and $u$ as in the summation. Since $a_{(T, \eta)}$ is $\alpha$-invariant we then have $\Phi\left(\delta^{\prime}\right)=\Phi(v \delta)=\Phi(u \delta)$ which clearly coincides with $\Phi(\delta)$. Thus the lemma is proved.

Finally, suppose that $(T, \eta) \in \mathcal{T}_{H}(G)$ and that $i(h, \eta): T^{\prime} \rightarrow T$ is defined over $\mathbf{R}$. Then the Haar measure $d t$ on $T(\mathbf{R})$ is transported via $i(h, \eta)$ to a Haar measure $d t^{\prime}$ on $T^{\prime}(\mathbf{R}) ; d t^{\prime}$ is independent of the choice of $h$. Also, we say that $\gamma^{\prime} \in T^{\prime}(\mathbf{R})_{\text {reg }}$ is not a norm if it is not in the image of the norm map from $\tilde{T}^{\prime}=\operatorname{Res}_{\mathbf{R}}^{\mathbf{C}} T^{\prime}$ to $T^{\prime}$, i.e. $\gamma^{\prime}$ does not lie in the identity component of $T^{\prime}(\mathbf{R})$. Then if $\gamma^{\prime}$ originates from $\gamma \in T(\mathbf{R})_{\text {reg }}$ via $(T, \eta), \gamma$ is not in the image of the norm from $\tilde{T}$ to $T$ (and conversely...).

We have not assumed that $\xi$ or $\tilde{\xi}$ is of "unitary type" [Sh3]. It is easily checked that there is a quasicharacter $\chi$ on $H(\mathbf{R})$ such that $\left|\chi\left(\gamma^{\prime}\right) \Lambda_{(T, \eta)}(\gamma) a_{(T, \eta)}(\delta)\right|=1$ if $\gamma^{\prime}$ originates from $\gamma=\delta \alpha(\delta)$ via $(T, \eta)$. We then define $\mathcal{C}_{\tilde{\xi}}(H(\mathbf{R}))$ to be the set of functions $f$ on $H(\mathbf{R})$ such that $f \chi$ belongs to $\mathcal{C}(H(\mathbf{R}))$, the Schwartz space of $H(\mathbf{R})$. As the notation indicates, this space does not depend on the choice of $\chi$. For $f \in \mathcal{C}_{\tilde{\xi}}(H(\mathbf{R}))$ the 
stable orbital integrals $\Phi_{f}^{\left(T^{\prime}, 1\right)}\left(\gamma^{\prime}, d t^{\prime}, d h\right), \gamma^{\prime} \in T^{\prime}(\mathbf{R}) \cap H_{\text {reg }}$ (cf. [Sh4] etc.) are well-defined. Thus

It remains now to recall the factor $\Delta_{(T, \eta)}$ from $L$-indistinguishability.

$$
\Delta_{(T, \eta)}=(-1)^{q(G, H)} \varepsilon(T, \eta) \Lambda_{(T, \eta)} \Delta_{(T, \eta)},
$$

where $q(G, H)$ is an integer, $(-1)^{q(G, H)}$ being inserted only for convenience, $\varepsilon(T, \eta)= \pm 1$ is defined implicitly, $\Lambda_{(T, \eta)}$ is as earlier in this section and ' $\Delta_{(T, \eta)}$ is a discriminant function (see [Sh4, §3] for further details).

\section{The matching theorem.}

TheOREM 7.1. Let $H$ be an endoscopic group for $(\tilde{G}, \alpha)$, with $L$-group ${ }^{L} H_{s}$ chosen as earlier. Suppose that $\tilde{\xi}:{ }^{L} H_{s} \hookrightarrow{ }^{L} \tilde{G}$ is an allowed embedding and that $\xi:{ }^{L} H_{s} \hookrightarrow{ }^{L} G$ is admissible (for L-indistinguishability). Then for each $f \in C_{c}^{\infty}(\tilde{G}(\mathbf{R}))$ there exists $f_{H} \in \bigodot_{\tilde{\xi}}(H(\mathbf{R}))$ such that:

$$
\Phi_{f_{H}}^{\left(T^{\prime}, 1\right)}\left(\gamma^{\prime}, d t^{\prime}, d h\right)=\left\{\begin{array}{l}
\Delta_{(T, \eta)}(\gamma) a_{(T, \eta)}(\delta) \Phi_{f}^{(T, \alpha, \kappa)}(\delta, d t, d \tilde{g}), \\
\text { if } \gamma^{\prime} \text { originates from } \gamma=\delta \alpha(\delta) \\
\text { via }(T, \eta) \in \mathcal{T}_{H}(G), \\
0 \quad \text { if } \gamma \text { is not a norm. }
\end{array}\right.
$$

Here it is assumed that $\gamma^{\prime}$ originates from regular elements in $G(\mathbf{R})$. Then $\gamma^{\prime}$ is regular in $H(\mathbf{R})$ [Sh2]; $T^{\prime}$ is the maximal torus containing $\gamma^{\prime}$. Recall that $\Delta_{(T, \eta)}$ depends on $\xi$ alone, that $a_{(T, \eta)}$ depends on both $\xi$ and $\tilde{\xi}$, and that $\Delta_{(T, \eta)}(\gamma) a_{(T, \eta)}(\delta)$ depends on $\tilde{\xi}$ alone... as long as $(T, \eta)$ and $\delta$ are fixed.

REMARK. We have used $C_{c}^{\infty}(\tilde{G}(\mathbf{R}))$ instead of the more natural $\mathcal{C}(\tilde{G}(\mathbf{R}))$ since the necessary analysis of "twisted $F_{f}$ " (cf. [Sh6]), for $f$ a Schwartz function, has not been carried out. Work of L. Clozel now in progress should settle this matter and allow us to replace $C_{c}^{\infty}(\tilde{G}(\mathbf{R}))$ by $\mathcal{C}(\tilde{G}(\mathbf{R}))$.

Proof of the theorem. Let $\gamma^{\prime} \in H(\mathbf{R})$. Suppose that $\gamma^{\prime}$ originates from $\gamma \in G_{\text {reg }}$ via $(T, \eta)$ and from $\bar{\gamma}$ via $(\bar{T}, \bar{\eta})$. Choose $\delta$ so that $\delta \alpha(\delta)=\gamma$. Write $\bar{\gamma}$ as $y \gamma y^{-1}$ and $\bar{\eta}$ as $\omega_{H} \circ \eta \circ$ ad $y^{-1}$, where $\omega_{H} \in \Omega\left(H, T_{H}\right) \subset$ $\Omega\left(G, T^{*}\right)$ and $y \in \mathfrak{A}(T)$ (cf. [Sh4, §3]). Then for $\bar{\delta}$ such that $\bar{\delta} \alpha(\bar{\delta})=\bar{\gamma}$ we may take $y \delta y^{-1}$, where $y \in G$ has been identified with its image in $\tilde{G}$ under the diagonal embedding. With this choice of $\bar{\delta}$ we have $a_{(\bar{T}, \bar{\eta})}(\bar{\delta})=$ $a_{(T, \eta)}(\delta)$. The relation between $\Delta_{(\bar{T}, \bar{\eta})}(\bar{\gamma})$ and $\Delta_{(T, \eta)}(\gamma)$ is described in [Sh4, $\S 3]$. 
For fixed $(T, \eta) \in \mathcal{T}_{H}(G)$ the function

$$
\gamma^{\prime} \rightarrow a_{(T, \eta)}(\delta) \Delta_{(T, \eta)}(\gamma) \Phi_{f}^{(T, \alpha, \kappa)}(\delta, d t, d \tilde{g}),
$$

if $\gamma^{\prime}$ originates from $\gamma=\delta \alpha(\delta)$ via $(T, \eta)$, is well-defined and invariant under $\mathfrak{U}\left(T^{\prime}\right)$. To prove this we invoke [Sh4, Propositions 2.4.5 and 3.1.2] and [Sh6, Lemma 4.3.2]. These results show that we have only to check that $a_{(T, \eta)}\left(\delta^{\omega}\right)=a_{(T, \eta)}(\delta)$ for $\omega$ an element of the Weyl group $\Omega(G, T)$ of $(G, T)$ which commutes with the Galois action on $T$ and "comes from $H$ " (i.e. $\omega \in \Omega_{0}(G, T) \cap \Omega^{(\kappa)}(G, T)$ as in [Sh4, Proposition 2.4.5]). But this invariance of $a_{(T, \eta)}$ follows easily from the fact that $\left\langle\tilde{\mu}_{0}, \lambda^{\vee}\right\rangle=0$ for $\lambda^{\vee}$ in the span of the roots of ${ }^{L} \tilde{T}^{0}$ in ${ }^{L} \tilde{H}_{s}^{0}$ (see the proof of Lemma 6.1).

Suppose now that we fix a "framework of Cartan subgroups [Sh3], [Sh4, §3.2]. Thus we have specified certain pairs $\left(T_{n}, \eta_{n}\right) \in \mathcal{T}_{H}(G)$ and embeddings $i_{n}=i\left(h_{n}, \eta_{n}\right): T_{n}^{\prime} \rightarrow T_{n}$ over $\mathbf{R}$; the set $\left\{T_{n}^{\prime}(\mathbf{R})\right\}$ provides a complete family of representatives, without redundancy, for the conjugacy classes of Cartan subgroups of $H(\mathbf{R})$. Given $\gamma^{\prime} \in T_{n}^{\prime}(\mathbf{R})$, set $\gamma=i_{n}\left(\gamma^{\prime}\right)$, and choose any $\delta$ such that $\delta \alpha(\delta)=\gamma$. Call $\gamma^{\prime} G$-regular if $\gamma$ is regular. Then for each $n$ we may consider the function on the $G$-regular elements of $T_{n}^{\prime}(\mathbf{R})$ given by

$$
\Phi_{n}\left(\gamma^{\prime}, d t^{\prime}, d h\right)=\left\{\begin{array}{l}
\varepsilon_{n} \hat{\Delta}_{\left(T_{n}, \eta_{n}\right)}(\gamma) a_{\left(T_{n}, \eta_{n}\right)}(\delta) \Phi_{f}^{\left(T_{n}, \alpha, \kappa_{n}\right)}(\gamma, d t, d \tilde{g}) \\
\text { if } \gamma^{\prime} \in T_{n}^{\prime}(\mathbf{R})^{0}, \\
0 \quad \text { if } \gamma^{\prime} \notin T_{n}^{\prime}(\mathbf{R})^{0},
\end{array}\right.
$$

where $\varepsilon_{n}= \pm 1$ (to be chosen), $\hat{\Delta}_{(T, \eta)}=\varepsilon\left(T_{n}, \eta_{n}\right) \Delta_{\left(T_{n}, \eta_{n}\right)}$ (i.e. $\hat{\Delta}_{(T, \eta)}$ is $\Delta_{(T, \eta)}$ with the $\varepsilon(T, \eta)$ removed), and $\kappa_{n}$ is the " $\kappa$ " associated to $\left(T_{n}, \eta_{n}\right)$. Note that $\left\{\left.\kappa_{n}\right|_{X_{*}\left(\left(\mathrm{~T}_{n}\right)_{\text {sc }}\right)}\right\}$ is exactly the set $\left\{\kappa_{n}\right\}$ from [Sh2, §7] and [Sh3, §2].

Suppose that we are able to show that there exists $f_{H} \in \mathcal{C}_{\tilde{\xi}}(H(\mathbf{R}))$ such that

$$
\Phi_{f_{H}}^{\left(T_{n}^{\prime}, 1\right)}\left(\gamma^{\prime}, d t^{\prime}, d h\right)=\left\{\begin{array}{l}
\Phi_{n}\left(\gamma^{\prime}, d t^{\prime}, d h\right) \text { if } \gamma^{\prime} \in T_{n}^{\prime}(\mathbf{R})^{0} \\
0 \quad \text { if } \gamma^{\prime} \notin T_{n}^{\prime}(\mathbf{R})^{0}
\end{array}\right.
$$

for all $G$-regular $\gamma^{\prime}$ in $T_{n}^{\prime}(\mathbf{R})$ and for all $n$ provided $\varepsilon_{m} \varepsilon_{n}=\varepsilon(m, n)$ whenever $T_{m}^{\prime}(\mathbf{R})$ and $T_{n}^{\prime}(\mathbf{R})$ are adjacent Cartan subgroups. Here $\varepsilon(m, n)$ is as defined in [Sh4, §3.5] (cf. [Sh2]). Then we shall take $\varepsilon_{n}=\varepsilon\left(T_{n}, \eta_{n}\right)$, so that by the results of $L$-indistinguishability (exp. [Sh4, §3.5]) there does exist $f_{H}$ satisfying $(*)$. It is then routine to verify that $f_{H}$ satisfies the statement of our theorem (see the first paragraph of this proof; similar arguments for $L$-indistinguishability are given in [Sh4, §3]). 
Returning to the condition on the existence of $f_{H}$, we have only to show that our family $\left\{\Phi_{n}(,),\right\}$ behaves like the family $\left\{\Phi_{n}\right\}$ of [Sh2, §9] (cf. [Sh4, §3.2]). The invariance and growth requirements being satisfied (clearly), only the "jump conditions" remain. Thus we need the jump formulas for the functions $\Psi_{(T, \eta)}$ :

$$
\gamma \rightarrow\left\{\begin{array}{l}
a_{(T, \eta)}(\delta) \hat{\Delta}_{(T, \eta)}(\gamma) \Phi^{(T, \alpha, \kappa)}(\delta, d t, d \tilde{g}) \text { if } \gamma \in T(\mathbf{R})_{\mathrm{reg}}^{0}, \\
0 \text { if } \gamma \in T(\mathbf{R})_{\mathrm{reg}}-T(\mathbf{R})^{0} .
\end{array}\right.
$$

These are contained essentially in the analysis of $\$ \S 4$ and 5 of [Sh6]. To be more precise, we seek analogues of Lemmas 5.2.2 and 5.2.5 of [Sh6], when " ' $\Delta_{T} \Phi^{\tau}$ " is replaced by the function above (with the necessary adjustment in the choice of positive system for the imaginary roots of $T$ used to define the factor $\left.\hat{\Delta}_{(T, \eta)}\right)$. The proof of the analogue of Lemma 5.2.2 is straightforward; because of notational complications we omit further details. Note that the " $\kappa$-signature" [Sh2] which appears depends only on $\left.\kappa\right|_{X_{*}\left(T_{\mathrm{sc}}\right)}$, i.e. the jump is indeed like that from $L$-indistinguishability. The analogue of Lemma 5.2 .5 will be stronger than the original statement, because we no longer need the assumption " $\kappa\left(\alpha^{\vee}\right)=1$ if (5.2.3) holds." We now have the exact analogue of [Sh2, Proposition 9.1] from $L$-indistinguishability. Indeed, let $\gamma_{0}$ be a semiregular element in $T(\mathbf{R})$ such that $\lambda\left(\gamma_{0}\right)=1$, where $\lambda$ is an imaginary root such that $\kappa\left(\lambda^{\vee}\right)=-1$. We wish to show that $\Psi_{(T, \eta)}$ is smooth on some neighborhood of $\gamma_{0}$. We may assume that $\gamma_{0} \in T(\mathbf{R})^{0}$. Fix $\delta_{0} \in T(\mathbf{R})^{0}$ such that $\delta_{0}^{2}=\gamma$. For $\gamma$ close to $\gamma_{0}$ choose $\delta$ close to $\delta_{0}$ such that $\delta^{2}=\gamma$. It will be sufficient to show that $\delta \rightarrow \Psi_{(T, \eta)}\left(\delta^{2}\right)$ is smooth near $\delta_{0}$. This follows immediately from Lemma 4.3.3 of [Sh6]. Note that this type of argument could not be used in the proof of Lemma 5.2.5 of [Sh6] because the "cross-section for the norm" was not smooth near $\gamma_{0}$.

We now complete the proof of Theorem 7.1 by the arguments already indicated.

8. The dual lifting. Again we fix an element of $\subseteq(\tilde{G}, \alpha)$ and choose a convenient representative $\left(s,{ }^{L} H_{s}\right)$ for this element, as in $\S 5$. Let $H_{s}$ be the corresponding endoscopic group. Since $H_{s}$ is, by definition, quasi-split over $\mathbf{R}$, the set $\Phi\left(H_{s}\right)$ [L2] consists of all equivalence classes of admissible homomorphisms $\phi: W \rightarrow{ }^{L} H_{s}$. Suppose that $\tilde{\xi}:{ }^{L} H_{s}^{-} \hookrightarrow{ }^{L} \tilde{G}$ is an allowed embedding. Then $\tilde{\xi}$ induces a map, also to be denoted $\tilde{\xi}$, from $\Phi\left(H_{s}\right)$ to $\Phi(\tilde{G})$; the image of the class of $\phi: W \rightarrow{ }^{L} H_{s}$ is the class of $\tilde{\phi}=\tilde{\xi} \circ \phi$ : $W \rightarrow{ }^{L} \tilde{G}$. It is easily checked that the image of $\Phi\left(H_{s}\right)$ in $\Phi(\tilde{G})$ is independent of the choice for $\tilde{\xi}$. By the remarks at the end of $\S 5$ it is also independent of the choice for $\left(s,{ }^{L} H_{s}\right)$. 
On the other hand, the automorphism $\alpha$ of $\tilde{G}$ has a standard dual [Sh7], again denoted $\alpha$ :

$$
\alpha((g, h) \times w)=(h, g) \times w, \quad h, g \in{ }^{L} G^{0}, w \in W .
$$

If $\phi: W \rightarrow{ }^{L} \tilde{G}$ is admissible then so is $\alpha \circ \phi: W \rightarrow^{L} \tilde{G}$. We write $\{\phi\}$ for the class of $\phi$ and $\{\phi\}^{\alpha}$ for the class of $\alpha \circ \phi$. Then $\Phi(\tilde{G})^{\alpha}=\{\{\phi\} \in \Phi(\tilde{G})$ : $\left.\{\phi\}^{\alpha}=\{\phi\}\right\}$.

For each element of $\subseteq(\tilde{G}, \alpha)$ we fix a representative $\left(s,{ }^{L} H_{s}\right)$ as before, and assume that each ${ }^{L} H_{s}$ has an allowed embedding $\tilde{\xi}$ in ${ }^{L} \tilde{G}$. Also, we will use $U_{H_{s}}$ to denote a union over the corresponding endoscopic groups.

THEOREM 8.1.

$$
\Phi(\tilde{G})^{\alpha}=\bigcup_{H_{s}} \tilde{\xi}\left(\Phi\left(H_{s}\right)\right) .
$$

Proof. Let $\phi: W \rightarrow{ }^{L} H_{s}$ be admissible. Set $\tilde{\phi}=\tilde{\xi} \circ \phi$. We may assume that $\phi\left(\mathbf{C}^{x} \times 1\right) \subset^{L} T^{0} \times \mathbf{C}^{x} \times 1$. Then clearly $\tilde{\phi}$ and $\alpha \circ \tilde{\phi}$ coincide on $\mathbf{C}^{x} \times 1$. We write $\phi(1 \times \sigma)$ as $n_{H} \times 1 \times \sigma \in{ }^{L} H_{s}$, and $\tilde{\xi}(1 \times 1 \times \sigma)$ as $\left(x m_{0}, m_{0}\right) \times 1 \times \sigma(\mathrm{cf}$. 5$)$. Then $\tilde{\phi}(1 \times \sigma)=\left(x n_{H} m_{0}, n_{H} m_{0}\right) \times 1 \times \sigma$ and

$$
\begin{aligned}
(\alpha \circ \tilde{\phi})(1 \times \sigma) & =\left(n_{H} m_{0}, x n_{H} m_{0}\right) \times 1 \times \sigma \\
& =\left(x^{-1}, x\right) \tilde{\phi}(1 \times \sigma)=g^{-1} \tilde{\phi}(1 \times \sigma) g
\end{aligned}
$$

where $g=(x, 1)$. Then clearly $\alpha \circ \tilde{\phi}=\operatorname{ad} g^{-1} \circ \tilde{\phi}$, and so $\tilde{\xi}(\Phi(H)) \subset$ $\Phi(\tilde{G})^{\alpha}$.

Suppose now that $\tilde{\phi}: W \rightarrow{ }^{L} \tilde{G}$ is admissible and that $\{\tilde{\phi}\}^{\alpha}=\{\tilde{\phi}\}$. Then it is sufficient to show that $\tilde{\phi}$ factors through some ${ }^{L} H_{s}$ (not necessarily among our fixed representatives) embedded (via an allowed embedding) in ${ }^{L} \tilde{G}$.

Let $S_{\tilde{\phi}}^{\alpha}=\left\{a \in{ }^{L} \tilde{G}^{0}: a \tilde{\phi}(w) a^{-1}=(\alpha \circ \tilde{\phi})(w), w \in W\right\}$. Then $S_{\tilde{\phi}}^{\alpha}$ is nonempty. If $a_{0}$ lies in $S_{\tilde{\phi}}^{\alpha}$ then so does $a_{0} z$, for $z \in \tilde{Z}^{W}$. In fact, then $S_{\tilde{\phi}}^{\alpha}=a_{0} S_{\tilde{\phi}}$, where $S_{\tilde{\phi}}$ is the centralizer of $\tilde{\phi}(W)$ in ${ }^{L} \tilde{G}^{0} \ldots$ recall that the results of [Sh4], with a little extra argument for the case $\tilde{\phi}$ unbounded, show that $S_{\tilde{\phi}}=S_{\tilde{\phi}}^{0} \tilde{Z}^{W}, S_{\tilde{\phi}}^{0}$ denoting the identity component in $S_{\tilde{\phi}}$. Choose $s=a_{0} \tilde{Z}^{W}$ contained in $S_{\tilde{\phi}}^{\alpha}$. Assume that $s$ consists of $\alpha$-semisimple elements (... we will prove below that such an $s$ exists). Then set ${ }^{L} H_{s}^{0}=$ $\left(\operatorname{Cent}_{\alpha}\left(s,{ }^{L} \tilde{G}^{0}\right)\right)^{0}$, and select ${ }^{L} B_{s}^{0},{ }^{L} T_{s}^{0}$ and $\{Y\}$ as in $\S 3$. To define a suitable action of $W$ on ${ }^{L} H_{s}^{0}$ we have just to give a homomorphism of 
$\operatorname{Gal}(\mathbf{C} / \mathbf{R})$ into $\operatorname{Aut}\left({ }^{L} H_{s}^{0},{ }^{L} B_{s}^{0},{ }^{L} T_{s}^{0},\{Y\}\right)$ such that $\sigma_{s}$, the image of $\sigma$, is "realized in $\operatorname{Cent}_{\alpha}\left(s,{ }^{L} \tilde{G}\right)=\operatorname{Cent}_{\alpha}\left(a_{0},{ }^{L} \tilde{G}\right)$ ". But

$$
\tilde{\phi}(1 \times \sigma)^{-1} a_{0} \alpha(\tilde{\phi}(1 \times \sigma))=a_{0} .
$$

Thus $\tilde{\phi}(1 \times \sigma)$ normalizes ${ }^{L} H_{s}^{0}$. We may write ad $\left.\tilde{\phi}(1 \times \sigma)\right|_{L_{H_{s}^{0}}}$ as $\omega \sigma_{s}$, where $\omega$ is an inner automorphism of ${ }^{L} H_{s}^{0}$ and $\sigma_{s} \in$ $\operatorname{Aut}\left({ }^{L} H_{s}^{0},{ }^{L} B_{s}^{0},{ }^{L} T_{s}^{0},\{Y\}\right)$. Note that $\sigma_{s}^{2}=1$ and is "realized in Cent ${ }_{\alpha}\left(s,{ }^{L} \tilde{G}\right)$ ". Using the associated $W$-action we form ${ }^{L} H_{s}$ and so obtain a representative $\left(s,{ }^{L} H_{s}\right)$ for an element of $\subseteq(\tilde{G}, \alpha)$. We claim that $\tilde{\phi}$ factors through ${ }^{L} H_{s}$. Thus, suppose that $\tilde{\xi}:{ }^{L} H_{s} \hookrightarrow{ }^{L} \tilde{G}$ is an allowed embedding. Then for each $w \in W, \tilde{\phi}(w)$ lies in $\operatorname{Cent}_{\alpha}\left(s,{ }^{L} \tilde{G}\right)$ and acts on ${ }^{L} H_{s}^{0}=\left(\operatorname{Cent}_{\alpha}\left(s,{ }^{L} \tilde{G}^{0}\right)\right)^{0}$ as an element $n(w) \times w$ of the image of ${ }^{L} H_{s}$ in ${ }^{L} \tilde{G}$. By definition, $n(w) \times w \in \operatorname{Cent}_{\alpha}\left(s,{ }^{L} \tilde{G}\right)$. Thus $\tilde{\phi}(w)=a(w)(n(w) \times$ $w)$, where $a(w) \in \operatorname{Cent}_{\alpha}\left(s,{ }^{L} \tilde{G}^{0}\right)$ centralizes ${ }^{L} H_{s}^{0}$. But then $a(w)$ lies in the center of ${ }^{L} H_{s}^{0}$. Hence $\tilde{\phi}$ factors through ${ }^{L} H_{s}$.

It remains now to show that $S_{\tilde{\phi}}^{\alpha}$ contains an $\alpha$-semisimple element. If we replace $\tilde{\phi}$ by ad $g \circ \tilde{\phi}, g \in{ }^{L} \tilde{G}^{0}$, then we must replace $S_{\tilde{\phi}}^{\alpha}$ by $\alpha(g) S_{\tilde{\phi}}^{\alpha} g^{-1}$. Therefore we may assume that $S_{\tilde{\phi}}^{\alpha}$ contains an element $\left(x^{-1}, 1\right), x \in{ }^{L} G^{0}$ (cf. Lemma 4.1). Then we write $\tilde{\phi}(w)$ as $\left(\phi_{1}(w), \phi_{2}(w)\right) \times w$ and obtain from $\left(x^{-1}, 1\right) \tilde{\phi}(w)(x, 1)=\alpha(\tilde{\phi}(w)), w \in W$, that $\phi_{1}(z \times 1)=\phi_{2}(z \times 1)$, $z \in \mathbf{C}^{x}$, and $\phi_{1}(1 \times \sigma)=x \phi_{2}(1 \times \sigma)$; also, $x$ lies in the centralizer $S_{0}$ of the image of the homomorphism $\hat{\phi}_{1}: w \rightarrow \phi_{1}(w) \times w$ of $W$ into ${ }^{L} G$. Write $x=x_{u} x_{s}$, where $x_{u} \in S_{0}$ is unipotent and $x_{s} \in S_{0}$ is semisimple. Then with the same $\phi_{1}$ and with $x_{s}$ in place of $x$ we can use the formulas above for $\tilde{\phi}$ to define $\tilde{\phi}_{0}: W \rightarrow{ }^{L} \tilde{G}$ such that $S_{\tilde{\phi}_{0}}^{\alpha}$ contains $\left(x_{s}^{-1}, 1\right)$. But $\tilde{\phi}_{0}$ is easily seen to be equivalent to $\tilde{\phi}$ because $x_{u}^{-1}$, being unipotent and fixed by $\hat{\phi}_{1}(W)$, can be written as $v\left(\hat{\phi}_{1}(1 \times \sigma) v\right)^{-1}, v \in \operatorname{Cent}\left(\phi_{1}\left(\mathbf{C}^{x} \times 1\right),{ }^{L} G^{0}\right)$. Since $\left(x_{s}^{-1}, 1\right)$ is $\alpha$-semisimple our proof of Theorem 8.1 is complete.

According to Langlands' functoriality principle this factoring of the $\alpha$-fixed parameters $\{\tilde{\phi}\}$ should be reflected in character theory. Let $\tilde{\phi} \in \Phi(\tilde{G})$ be $\alpha$-fixed (we now drop the \{\} from the notation for parameters). Then the $L$-packet $\Pi_{\tilde{\phi}}$ consists of a single infinitesimal equivalence class of irreducible admissible representations fixed by the automorphism $\alpha: \tilde{G}(\mathbf{R}) \rightarrow \tilde{G}(\mathbf{R})(\ldots$ this is easily checked, see also [C1]). Thus the twisted character $\chi_{\tilde{\phi}}^{\alpha}$ of $\Pi_{\tilde{\phi}}$ is well-defined up to sign (see [C1] for a detailed discussion, especially concerning the question of signs). Assume that $\tilde{\phi}$ is bounded, i.e. if $\tilde{\phi}(w)=\tilde{\phi}_{0}(w) \times w, w \in W$, then $\tilde{\phi}_{0}(W)$ is bounded. Then $\chi_{\tilde{\phi}}^{\alpha}$ is tempered [C1, Theorem 5.12]. On the other hand, suppose that $\tilde{\phi}$ is the lift of $\phi \in \Phi(H)$, in the sense afforded by Theorem 
8.1. Then $\phi$ is essentially bounded, so that the $L$-packet $\Pi_{\phi}$ consists of essentially tempered (equivalence classes of) representations. Thus $\chi_{\phi}=$ $\Sigma_{\pi \in \Pi_{\phi}} \chi_{\pi}, \chi_{\pi}$ denoting the ordinary character of $\pi$, is a stable essentially tempered distribution on $H(\mathbf{R})$ [Sh1, Lemma 5.2].

Theorem 7.1 provides a correspondence $\left(f, f_{H}\right)$ between $C_{c}^{\infty}(G(\mathbf{R}))$ and $\bigodot_{\tilde{\xi}}(H(\mathbf{R}))$. As mentioned already, an adequate analysis of the "twisted $F_{f}$ transform" would provide a correspondence between $\mathcal{C}(\tilde{G}(\mathbf{R}))$ and $\bigodot_{\tilde{\xi}}(H(\mathbf{R}))$; it would also give a dual lifting of stable tempered distributions on $H(\mathbf{R})$ to twisted-invariant tempered distributions on $\tilde{G}(\mathbf{R})$, with eigendistributions mapping to eigendistributions (see [Sh4, §4] for the analogous arguments in the case of $L$-indistinguishability). Nevertheless, with the correspondence of Theorem 7.1 we can define (Lift $\left.\chi_{\phi}\right)(f)=\chi_{\phi}\left(f_{H}\right)$, $f \in C_{c}^{\infty}(\tilde{G}(\mathbf{R}))$. Writing $\chi_{\phi}\left(f_{H}\right)$ as $\int_{H(\mathbf{R})} f_{H}(h) \chi_{\phi}(h) d h$, and applying the Weyl Integration Formula, the matching theorem and the twisted analogue of the Weyl Integration Formula, we find that Lift $\chi_{\phi}$ is a twistedinvariant distribution on $\tilde{G}(\mathbf{R})$ represented by a function explicitly computed in terms of $\chi_{\phi}$. Moreover, this function transforms under the center of the universal enveloping algebra of $\tilde{G}(\mathbf{C})$ according to the infinitesimal character of $\chi_{\tilde{\phi}}^{\alpha}$. We may therefore ask if Lift $\chi_{\phi}$ coincides with $\chi_{\tilde{\phi}}^{\alpha}$ up to a constant (depending only on $G$ and $H$, once the sign for $\chi_{\tilde{\phi}}^{\alpha}$ has been suitably fixed). According to [C1] with some minor additional arguments, this is true if $H=G$; recall that we are assuming that $\tilde{\phi}$ is bounded, so that $\phi$ is an essentially bounded parameter. Work of L. Clozel now in progress should provide the answer to our question for the case $H \neq G$.

\section{REFERENCES}

[C1] L. Clozel, Changement de base pour les representations tempérées des groupes reductifs réels, Ann. Sci. Ec. Norm. Sup., $4^{\mathrm{e}}$ serie, t. 15 (1982), 45-115.

[L1] R. Langlands, Stable conjucacy: definitions and lemmas Canad. J. Math., XXXI, No. 4 (1979), 700-725.

[L2] _ On the classification of irreducible representations of real algebraic groups, preprint (1973).

[L3] Les débuts d'une formule des traces stables, Publ. Math. Univ. Paris VII, vol. 13 (1983).

[Sh1] D. Shelstad, Characters and inner forms of a quasi-split group over $R$, Compositio Math., 39 (1979), 11-45.

[Sh2] '_ Orbital integrals and a family of groups attached to a real reductive group, Ann. Sci. Ec. Norm. Sup., $4^{\mathrm{e}}$ serie, t. 12 (1979), 1-31.

[Sh3] __ Embeddings of L-groups, Canad. J. Math., 33 (1981), 513-558.

[Sh4]

[Sh5] , L-indistinguishability for real groups, Math. Ann., 259 (1982), 385-430. , Orbital integrals, endoscopic groups and L-indistinguishability for real groups, to appear in Publ. Math. Univ. Paris VII. 
[Sh6] _ Base Change and a Matching Theorem for Real Groups, in Springer Lecture Notes, Vol. 880 (1982), pp. 425-482.

[Sh7] _ A generalization of endoscopic groups, (in preparation).

Received April 20, 1982. Supported by NSF Grants MCS 79-01712, MCS 81-02392 and MCS 81-08814(A01).

RUTGERS UNIVERSITY

NEWARK, NJ 07102 


\title{
PACIFIC JOURNAL OF MATHEMATICS EDITORS
}

\author{
DONALD BABBITT (Managing Editor) \\ University of California \\ Los Angeles, CA 90024 \\ Hugo Rossi \\ University of Utah \\ Salt Lake City, UT 84112 \\ C. C. MOORE and Arthur Ogus \\ University of California \\ Berkeley, CA 94720
}

\author{
J. Dugundir \\ Department of Mathematics \\ University of Southern California \\ Los Angeles, CA 90089-1113
}

R. FINN and H. SAMELSON

Stanford University

Stanford, CA 94305

ASSOCIATE EDITORS
R. ARENS
E. F. BECKENBACH
B. H. NeumanN
F. WOLF
K. YosHIDA (1906-1982)

\section{SUPPORTING INSTITUTIONS}

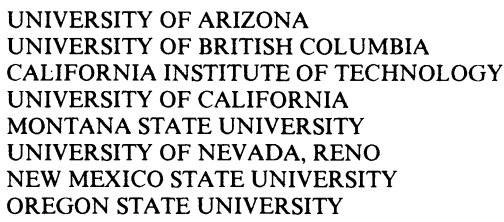

\author{
UNIVERSITY OF OREGON \\ UNIVERSITY OF SOUTHERN CALIFORNIA \\ STANFORD UNIVERSITY \\ UNIVERSITY OF HAWAII \\ UNIVERSITY OF TOKYO \\ UNIVERSITY OF UTAH \\ WASHINGTON STATE UNIVERSITY \\ UNIVERSITY OF WASHINGTON
}

The Supporting Institutions listed above contribute to the cost of publication of this Journal, but they are not owners or publishers and have no responsibility for its content or policies.

Mathematical papers intended for publication in the Pacific Journal of Mathematics should be in typed form or offset-reproduced (not dittoed), double spaced with large margins. Please do not use built up fractions in the text of the manuscript. However, you may use them in the displayed equations. Underline Greek letters in red, German in green, and script in blue. The first paragraph must be capable of being used separately as a synopsis of the entire paper. In particular it should contain no bibliographic references. Please propose a heading for the odd numbered pages of less than 35 characters. Manuscripts, in triplicate, may be sent to any one of the editors. Please classify according to the scheme of Math. Reviews, Index to Vol. 39. Supply name and address of author to whom proofs should be sent. All other communications should be addressed to the managing editor, or Elaine Barth, University of California, Los Angeles, California 90024.

There are page-charges associated with articles appearing in the Pacific Journal of Mathematics. These charges are expected to be paid by the author's University, Government Agency or Company. If the author or authors do not have access to such Institutional support these charges are waived. Single authors will receive 50 free reprints; joint authors will receive a total of 100 free reprints. Additional copies may be obtained at cost in multiples of 50 .

The Pacific Journal of Mathematics is issued monthly as of January 1966. Regular subscription rate: \$132.00 a year (6 Vol., 12 issues). Special rate: $\$ 66.00$ a year to individual members of supporting institutions.

Subscriptions, orders for numbers issued in the last three calendar years, and changes of address should be sent to Pacific Journal of Mathematics, P.O. Box 969, Carmel Valley, CA 93924, U.S.A. Old back numbers obtainable from Kraus Periodicals Co., Route 100, Millwood, NY 10546.

The Pacific Journal of Mathematics ISSN 0030-8730 is published monthly by the Pacific Journal of Mathematics at P.O. Box 969, Carmel Valley, CA 93924. Application to mail at Second-class postage rates is pending at Carmel Valley, California, and additional mailing offices. Postmaster: Send address changes to Pacific Journal of Mathematics, P. O. Box 969, Carmel Valley, CA 93924.

PUBLISHED BY PACIFIC JOURNAL OF MATHEMATICS, A NON-PROFIT CORPORATION

Copyright $(1984$ by Pacific Journal of Mathematics 


\section{Pacific Journal of Mathematics}

Vol. 110, No. $2 \quad$ October, 1984

Robert A. Bekes, The range of convolution operators $\ldots \ldots \ldots \ldots \ldots 257$

Dennis K. Burke and Sheldon Davis, Subsets of ${ }^{\omega} \omega$ and generalized metric

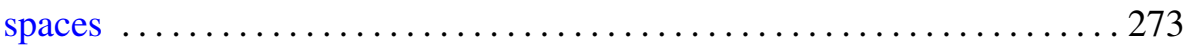

Giovanni Emmanuele, A remark on a paper: "Common fixed points of nonexpansive mappings by iteration" $\ldots \ldots \ldots \ldots \ldots \ldots \ldots \ldots \ldots 283$

I. Erdélyi and Sheng-Wang Wang, On strongly decomposable operators . . . 287

Gerhard Gierz, Injective Banach lattices with strong order units . . . . . . . 297

Maurizio Letizia, Quotients by complex conjugation of nonsingular quadrics and cubics in $\mathbf{P}_{\mathbf{C}}^{3}$ defined over $\mathbf{R} \ldots \ldots \ldots \ldots \ldots \ldots \ldots \ldots \ldots \ldots$

P. H. Maserick and Franciszek Hugon Szafraniec, Equivalent definitions

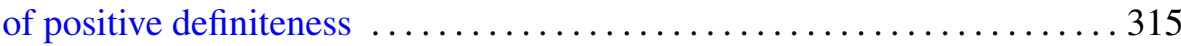

Costel Peligrad and S. Rubinstein, Maximal subalgebras of $C^{*}$-crossed

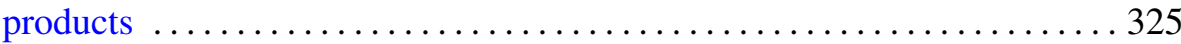

Derek W. Robinson and Sadayuki Yamamuro, Hereditary cones, order

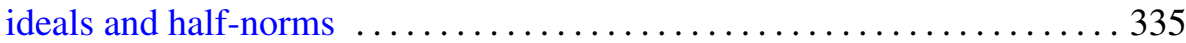

Derek W. Robinson and Sadayuki Yamamuro, The Jordan decomposition

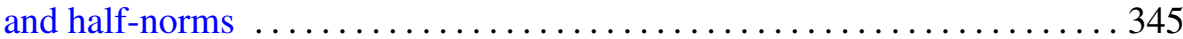

Richard Rochberg, Interpolation of Banach spaces and negatively curved

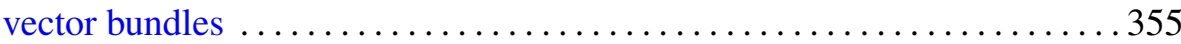

Dale Rolfsen, Rational surgery calculus: extension of Kirby's theorem 377

Walter Iaan Seaman, Helicoids of constant mean curvature and their Gauss maps

Diana Shelstad, Endoscopic groups and base change $\mathbf{C} / \mathbf{R}$

Jerrold Norman Siegel and Frank Williams, Numerical invariants of homotopies into spheres

Alladi Sitaram, Some remarks on measures on noncompact semisimple Lie groups

Teruhiko Soma, Atoroidal, irreducible 3-manifolds and 3-fold branched coverings of $S^{3}$

Jan de Vries, On the $G$-compactification of products

Hans Weber, Topological Boolean rings. Decomposition of finitely additive set functions 